\title{
Hydrophobization of cellulose nanocrystals for aqueous colloidal suspensions and gels
}

Rinat Nigmatullin, Marcus A. Johns, Juan C. Muñoz-García, Valeria Gabrielli, Julien Schmitt, Jesús Angulo, Yaroslav Z. Khimyak, Janet Lesley Scott, Karen J. Edler, and Stephen J. Eichhorn

Biomacromolecules, Just Accepted Manuscript • DOI: 10.1021/acs.biomac.9b01721 • Publication Date (Web): 25 Jan 2020

Downloaded from pubs.acs.org on January 30, 2020

\section{Just Accepted}

"Just Accepted" manuscripts have been peer-reviewed and accepted for publication. They are posted online prior to technical editing, formatting for publication and author proofing. The American Chemical Society provides "Just Accepted" as a service to the research community to expedite the dissemination of scientific material as soon as possible after acceptance. "Just Accepted" manuscripts appear in full in PDF format accompanied by an HTML abstract. "Just Accepted" manuscripts have been fully peer reviewed, but should not be considered the official version of record. They are citable by the Digital Object Identifier (DOI®). "Just Accepted" is an optional service offered to authors. Therefore, the "Just Accepted" Web site may not include all articles that will be published in the journal. After a manuscript is technically edited and formatted, it will be removed from the "Just Accepted" Web site and published as an ASAP article. Note that technical editing may introduce minor changes to the manuscript text and/or graphics which could affect content, and all legal disclaimers and ethical guidelines that apply to the journal pertain. ACS cannot be held responsible for errors or consequences arising from the use of information contained in these "Just Accepted" manuscripts. 


\title{
Hydrophobization of cellulose nanocrystals for aqueous colloidal suspensions and gels
}

\author{
Rinat Nigmatullin ${ }^{1 *}$, Marcus A. Johns ${ }^{1}$, Juan C. Muñoz-García ${ }^{2}$, Valeria Gabrielli², Julien \\ Schmitt ${ }^{3,4}$, Jesús Angulo ${ }^{2 \dagger}$, Yaroslav Z. Khimyak ${ }^{2}$, Janet L. Scott ${ }^{3}$, Karen J. Edler ${ }^{3}$, Stephen J. \\ Eichhorn $^{1 *}$
}

1. Department of Aerospace Engineering, Bristol Composites Institute (ACCIS), University of Bristol, Bristol, BS8 1TR, UK.

2. School of Pharmacy, University of East Anglia, Norwich Research Park, Norwich, NR4 7TJ, UK.

3. Department of Chemistry, University of Bath, Claverton Down, Bath, BA2 7AY, UK.

4. LSFC - Laboratoire de Synthèse et Fonctionnalisation des Céramiques UMR 3080 CNRS /

Saint-Gobain CREE, Saint-Gobain Research Provence, 550 avenue Alphonse Jauffret, Cavaillon, France.

\author{
Author Information \\ Corresponding authors: \\ Stephen J. Eichhorn: $\underline{\text { s.j.eichhorn@bristol.ac.uk }}$ \\ Rinat Nigmatullin: $\underline{\text { n17541@,bristol.ac.uk }}$
}


KEYWORDS. Cellulose nanocrystals, hydrophobization, surface activity, rheological properties.

\begin{abstract}
ADSTRACT. Surface hydrophobization of cellulose nanomaterials has been used in the development of nanofiller-reinforced polymer composites and formulations based on Pickering emulsions. Despite well-known effect of hydrophobic domains on self-assembly or association of water-soluble polymer amphiphiles, very few studies have addressed the behavior of hydrophobized cellulose nanomaterials in aqueous media. In this study, we investigate the properties of hydrophobized cellulose nanocrystals (CNCs) and their self-assembly and amphiphilic properties in suspensions and gels. CNCs of different hydrophobicity were synthesized from sulfated CNCs by coupling primary alkylamines of different alkyl chain lengths (6, 8 and 12 carbon atoms). The synthetic route permitted the retention of surface charge, ensuring good colloidal stability of hydrohobized CNCs in aqueous suspensions. We compare surface properties (surface charge, Zeta-potential), hydrophobicity (water contact angle, microenvironment probing using pyrene fluorescence emission) and surface activity (tensiometry) of different hydrophobized CNCs and hydrophilic CNCs. Association of hydrophobized CNCs driven by hydrophobic effects is confirmed by X-ray scattering (SAXS) and autofluorescent spectroscopy experiments. As a result of $\mathrm{CNC}$ association, CNCs suspensions/gels can be produced with a wide range of rheological properties depending on the hydrophobic/hydrophilic balance. In particular, sol-gel transitions for hydrophobized CNCs occur at lower concentrations then hydrophilic CNCs and more robust gels are formed by hydrophobized CNCs. Our work illustrates that amphiphilic CNCs can complement associative polymers as modifiers of rheological properties of water-based systems.
\end{abstract}




\section{Introduction}

Advanced functional materials and devices based on nanomaterials capitalize on the unique properties of the nanoparticles used as building elements. ${ }^{1,2}$ A vast diversity of potential applications has generated demand for nanoparticles of different shapes (spheres, rods, fibrils, platelets, etc.), sizes, and chemical compositions. Progress in the chemical synthesis of nanoparticles has been complemented by their isolation or construction from materials of biological origin. ${ }^{3,4}$ These latter approaches provide sustainable routes for nanoparticle generation. In this respect, nanomaterial isolation from various cellulose-rich biomass (wood, plants, algae, bacterial biomass, etc.) becomes an established approach to generate highly crystalline nanoparticles of high aspect ratio with diameters from 5 to $100 \mathrm{~nm}$ and length from tens to several hundred nanometers. ${ }^{5,6}$ The high crystallinity of cellulose nanomaterials (CNMs) defines their exceptional strength and stiffness. Also, CNM morphology, reactivity and topochemistry provide a versatile platform for advanced functional materials.

The success in CNM utilization as building blocks largely depends on their directed or self-assembly in isolation, or in combination with other components into specific structures. ${ }^{7-9}$ Assembly of nanoparticles requires certain chemical motifs on their surface. For example, sulfated cellulose nanocrystals (CNCs) form stable chiral nematic liquid crystalline phases ${ }^{10}$, whilst low surface charge CNCs tend to assemble at the liquid-liquid interface due to the hydrophobic, or hydrophilic nature of their different crystal faces. ${ }^{11}$ Therefore, surface modification or functionalization is an important, very often indispensable, step towards functional nanomaterials. On the molecular level CNMs contain reactive hydroxyl groups, which enable changes in surface properties via very diverse and well-established chemical pathways. ${ }^{12,13}$ 
Hydrophobization of CNMs has been well-documented in the literature. Various chemical routes have been employed for functionalization of CNM surfaces with hydrophobic domains via covalent binding. CNM hydrophobization has been conducted via silylation using alkyldimethylchlorosilanes with various lengths of alkyl groups ${ }^{14-16}$, esterification with acyl chlorides $^{17,18}$, and urethanization using hydrophobic isocyanates ${ }^{19,20}$. These one-step modification methods involve water-sensitive reactants and, therefore, require organic solvents for the reactions. Moreover, for these chemical routes there is a risk of a reduction in the crystallinity of the cellulose, or even obtaining soluble products if the degree of functionalization is too high. Graft copolymerization has also been used for CNM hydrophobization. ${ }^{21,22}$ Water-based hydrophobization of anionic CNMs (sulfated CNCs, TEMPO-oxidized CNMs) has been achieved via ionic interactions with cationic surfactants containing long alkyl groups. ${ }^{23-34}$ This is a simple approach for the hydrophobization of CNMs. However, binding is reversible, and modifying surfactants can be released from the CNM surface. Reductive amination is an another modification route for CNMs, which can be conducted in aqueous media. ${ }^{35}$ In this case, CNMs are activated by periodate oxidation leading to partial transformation into dialdehyde cellulose, which is followed by a reaction of the formed aldehyde groups with primary amines. Unlike ionic binding, reductive amination results in covalent binding of the modifying agent, which can be a hydrophobic amine. For example, modification with butylamine isomers using this route enabled isolation of hydrophobized CNCs from Kraft pulp without acid hydrolysis. ${ }^{36,37}$ Pre-activation of CNMs can also be omitted since cellulose chains contain aldehyde groups at the reducing end. ${ }^{38} \mathrm{CNM}$ activation for covalent binding of hydrophobic amines has been achieved by coating CNCs with 
tannic acid as a reactive primer. ${ }^{39}$ For this approach all modifications steps were also conducted in aqueous media.

The interest in hydrophobic derivatives of CNMs has mainly been driven by the potential applications in two fields: polymer nanocomposites and emulsion stabilization. The exceptional strength and stiffness of CNMs have also raised great expectations for the development of polymer nanocomposites. ${ }^{40}$ However, most synthetic polymers are hydrophobic materials, which results in poor wettability of CNMs and weak adhesion with the polymer matrix. Thus, surface hydrophobization has been considered as means for improving the compatibility between nanocomposite components. ${ }^{18,19,32,41-44}$ Emulsion stabilization by unmodified CNMs has also given impetus for functionalization of CNMs with hydrophobic domains for the enhancement of emulsifying properties. ${ }^{11,17,28,29,31,36-38}$ However, the literature lacks studies focused on hydrophobized CNMs in aqueous systems, despite the fact that water soluble polymers with moderate contents of hydrophobic moieties are widely used as viscosity modifiers in various aqueous industrial formulations such as paints, pharmaceuticals, cosmetics, foods, etc. ${ }^{45-47}$ These applications are based on the ability of such polymers to form a transient network due to reversible association between the hydrophobic groups. Recently it was demonstrated that properly adjusted hydrophobization of charged CNCs resulted in derivatized CNCs forming stable aqueous suspensions. ${ }^{21,48}$ Such associative CNCs undergo sol to gel transitions in aqueous suspensions at significantly lower concentrations compared with their hydrophilic counterparts. Gels of hydrophobized exhibited higher viscosities and stronger elastic responses, which was attributed to the formation of transient networks driven by hydrophobic effects. With these hydrophobized CNCs, hydrophobic effects can be utilized for the design of hybrid systems consisting of 
nanoparticles and other water soluble polymers as it was demonstrated in combinations with starch, a helical polysaccharide ${ }^{48}$, and thermally responsive hydroxypropyl methyl cellulose. ${ }^{49}$ Thus hydrophobized CNMs have a potential to complement a group of associative materials for their use in water based systems.

This study reports the properties of aqueous colloidal systems with hydrophobized CNCs, with a focus on the impact of hydrophobic effects on CNCs association and gelation in aqueous media. A synthetic route based on reductive amination was adopted for $\mathrm{CNC}$ hydrophobization with the intention to use water-based modification leading to covalent binding of hydrophobic groups of various length to the $\mathrm{CNC}$ surface. Detailed characterization of these hydrophobized CNCs in aqueous suspensions was obtained compared to the parent hydrophilic CNCs by surface tensiometry, electrophoretic light scattering, fluorescence anisotropy, autofluorescent spectroscopy, small-angle X-ray scattering (SAXS), oscillatory rheology and steady-shear viscometry. We demonstrate that self-association driven by hydrophobic effects induces sol-gel transformation at lower concentrations of hydrophobized CNCs and leads to the formation of more robust gels. Thus, amphiphilic derivatives of CNCs, or other CNMs, could further expand the selection of associative polymers to offer more flexibility in modifications of rheological properties of water-based systems.

\section{Experimental Methods}

Chemical modification of CNCs. The CNC surfaces were modified by binding alkylamines of different chain length, hexylamine $\left(\mathrm{C}_{6}-\mathrm{CNCs}\right)$, octylamine $\left(\mathrm{C}_{8}-\mathrm{CNCs}\right)$, and dodecylamine $\left(\mathrm{C}_{12}\right.$-CNCs) according to a procedure previously described. ${ }^{49}$ Firstly, the $\mathrm{CNC}$ surfaces were 
activated by oxidation in aqueous suspension (1.6 wt.\%) using sodium periodate (1.68 mmol of $\mathrm{NaIO}_{4}$ per $1 \mathrm{~g}$ of $\mathrm{CNC}$ ) at room temperature for 48 hours. After oxidation, the $\mathrm{CNC}$ suspension was dialyzed against deionized (DI) water for 24 hours using a cellulose membrane with a molecular cutoff of $\sim 14 \mathrm{kDa}$. Alkylamines ( $7.7 \mathrm{mmol}$ per $1 \mathrm{~g}$ of CNCs) were added to the purified suspension of oxidized CNCs. Reaction of oxidized CNCs with alkylamines was firstly conducted at $45^{\circ} \mathrm{C}$ for 3 hours, and then for a further $21 \mathrm{~h}$ at room temperature after adding $\mathrm{NaBH}_{3} \mathrm{CN}$ (40 $\mathrm{mM}$ ). Modified $\mathrm{CNCs}$ were purified using centrifugation and a 2 wt.\% $\mathrm{NaCl}$ solution in an iso-propanol/water mixture $(50 / 50 \mathrm{v} / \mathrm{v})$ as a washing solvent. Finally, modified CNCs were re-dispersed and dialyzed in and against DI water. The purified modified CNC suspensions were concentrated by allowing water evaporation through the dialysis membrane. To evaluate non-covalent binding of alkylamines to the CNCs' surface, the oxidation step was omitted, and CNC suspensions were directly treated with octylamine. Reagent ratio and purification steps were the same as in the preparation of covalently modified CNCs. All modified CNCs were stored as never-dried materials.

Nuclear magnetic resonance (NMR) spectroscopy. Solid-state NMR experiments were performed using a Bruker Avance III spectrometer equipped with a $4 \mathrm{~mm}$ triple resonance probe operating at frequencies of $300.13 \mathrm{MHz}\left({ }^{1} \mathrm{H}\right)$ and $75.47 \mathrm{MHz}\left({ }^{13} \mathrm{C}\right) . \mathrm{C}_{6}-\mathrm{CNC}, \mathrm{C}_{8}-\mathrm{CNC}$ and $\mathrm{C}_{12}$-CNC powders were tightly packed in an $80 \mu \mathrm{L}$ rotor and spun at a MAS rate of $12 \mathrm{kHz} .{ }^{1} \mathrm{H}-{ }^{13} \mathrm{C}$ CP/MAS NMR spectra (referenced with respect to TMS) were acquired at room temperature using 20k scans, a recycle delay of $10 \mathrm{~s}$ and a contact time of $1 \mathrm{~ms}$. It should be noted that it has been previously shown that ${ }^{1} \mathrm{H}-{ }^{13} \mathrm{C}$ CP-MAS NMR spectra of cellulose can be considered quantitative for $\mathrm{CP}$ contact times larger than $600 \mu \mathrm{s} .{ }^{50} \mathrm{CNC}$ specific surface area and degree of 
functionalisation was calculated from the peaks of C6 located in the interior and surface domains as described in SI.

Characterization of surface properties of CNCs. The content of sulfate groups on the surface of the CNCs was determined by conductometric titration ${ }^{51}$ using $20 \mathrm{ml}$ of a $\mathrm{CNC}$ suspension with a concentration of $\sim 3 \mathrm{mg} \mathrm{ml}^{-1}$. A $1.5 \mathrm{mM} \mathrm{NaOH}$ solution was used as the titrant. The conductivity values were corrected for dilution effects. The zeta potential of CNCs were measured with a Zetasizer Nano ZS (Malvern Instruments Ltd.) using $0.5 \mathrm{mg} \mathrm{mL}^{-1} \mathrm{CNC}$ suspensions in DI water. Zeta potential was estimated as an average of 15 measurements. Water contact angle measurements were conducted to estimate hydrophobicity of CNCs. Films of CNCs were prepared by drying $1 \mathrm{wt} . \%$ suspensions on glass slides. The automatic dispenser of a DSA100 drop shape analyzer (Krüss, Germany) was used to inject a droplet of known volume of $2 \mu \mathrm{L}$ on a substrate surface. ADVANCE software (Krüss, Germany) was used to analyze images of sessile drops and calculate static water contact angles.

Characterization of CNC suspensions. Surface tension of CNC aqueous suspensions was measured using an advanced surface tensiometer K100 (Krüss, Germany) equipped with a standard measuring probe (PL01) and by the Wilhelmy plate method. Measurements at different CNC concentrations were performed by automatic serial dilutions of CNC suspensions with an initial concentration of $7000 \mathrm{mg} \mathrm{L}^{-1}$ using two micro dispensers (DS0810). Pyrene fluorescence emission was used for probing the microenvironment in CNC suspensions. $100 \mu \mathrm{L}$ aliquots of $0.4 \mathrm{mM}$ pyrene solution in ethanol were dispensed into $7 \mathrm{ml}$ glass vials and allowed to dry at room temperature in the dark. $4 \mathrm{ml}$ of $\mathrm{CNC}$ suspensions with different concentrations were added to the 
vials resulting in a $10^{-5} \mathrm{M}$ pyrene concentration. The fluorescence emission spectra of pyrene probe at various $\mathrm{CNC}$ concentrations was recorded from 340 to $500 \mathrm{~nm}$ with $334 \mathrm{~nm}$ excitation wavelength by a FluoroMax-4 fluorescence spectrometer (HORIBA Instruments) The slit settings for excitation and emission were 2 and $1 \mathrm{~nm}$, respectively.

SAXS measurements. Suspensions of unmodified and modified CNCs at different concentrations (ranging from 1 to $10 \mathrm{wt} . \%$ for unmodified CNCs, 0.1 to $6 \mathrm{wt} . \%$ for $\mathrm{C} 8-\mathrm{CNCs}$ ), and suspensions in the presence of $0.1 \mathrm{M} \mathrm{KCl}$ were loaded in $1.5 \mathrm{~mm}$ diameter capillaries, sealed and measured using small angle X-ray scattering (SAXS). Part of the measurements was done at the I22 beamline of the Diamond Light Source (Didcot, Oxfordshire), operating at a wavelength $\lambda=1 \AA$ ( $E=12.4$ $\mathrm{keV}$ ), giving the following q-range: $4 \cdot 10^{-2}<\mathrm{q}<0.25 \AA^{-1}$. The data were collected using a Pilatus P3-2M (Silicon hybrid pixel detector, DECTRIS) averaging 10 frames of $100 \mathrm{~ms}$ exposure time each. Complementary measurements were done using a SAXSLab Ganesha 300XL instrument (SAXSLAB, ApS, Skovlunde, Denmark), operated at a wavelength $\lambda=1.54 \AA$ and equipped with a moveable Pilatus $300 \mathrm{~K} 2 \mathrm{D}$ detector. A similar q-range as Diamond $\left(4 \cdot 10^{-2}<\mathrm{q}<0.25 \AA^{-1}\right)$ was obtained by merging the patterns obtained at 3 different sample-detector distances and recorded for $1800 \mathrm{~s}, 3600 \mathrm{~s}$ and $7200 \mathrm{~s}$ respectively. For both I22 and Ganesha data, signals of the solvent and capillary were subtracted, and Lupolen was used for an absolute scaling calibration.

SAXS patterns were fitted using a model of interacting stiff rods. The rods are characterized by an elliptical cross-section of minor and major radii $R_{\min }$ and $R_{\max }$ (both in $\mathrm{nm}$ ) respectively and a length $L$ (in $\mathrm{nm}$ ). Interactions between CNCs were modelled using the PRISM model which depends of the strength of interaction via the so-called "excluded volume parameter" $v_{\mathrm{RPA}}(>0$ for repulsive interaction between $\mathrm{CNC}$ ) and a "hard-sphere" radius $R_{\mathrm{cq}}\left(\geq R_{\max }\right)$, corresponding to the 
section radius along the rods which is not accessible to any other rod. A full description of this model and its use for TEMPO-oxidized cellulose nanofibrils has previously been reported ${ }^{52}$.

Rheological measurements. A Discovery HR-1 rotational rheometer (TA Instrument) operating with a stainless steel cone plate geometry (dimeter $40 \mathrm{~mm}$, angle $4^{\circ}$ ) and a Peltier plate for temperature control was used for the rheological measurements of the CNC suspensions and gels. An isothermal dynamic amplitude sweep was performed to determine the linear viscoelastic (LVE) region and confirm that $1.5 \%$ strain was inside the LVE region for all tested samples. Frequency sweeps were conducted in strain-controlled mode at $1.5 \%$ strain for an angular frequency range from 0.4 to $100 \mathrm{rad} \mathrm{s}^{-1}$. Steady-state shear viscosity was measured within a shear rate range of 0.01 to $100 \mathrm{~s}^{-1}$.

\section{Results and Discussion}

\section{Surface Modification of CNCs}

With the aim to use water as a medium for $\mathrm{CNC}$ modification, a reductive amination of aldehyde groups with primary amines has been selected as the chemical route for hydrophobization. CNCs produced by hydrolysis in concentrated solutions of sulfuric acid are, in fact, functionalized nanoparticles due to the formation of sulfate half-ester groups on the CNC surface. As a result, the covalent linkage of alkylamine to the CNC surface can be accompanied with ionic binding of alkylamines due to interaction between sulfate and amine groups. Although ionic binding has been used for $\mathrm{CNC}$ hydrophobization in some applications ${ }^{23-34}$, it is reversible and potentially can lead to undesired or even harmful release of a cationic modifier. Therefore, the procedure for CNC modification was designed to minimize ionic binding. To avoid the establishment of ionic 
interactions between protonated amines, the $\mathrm{pH}$ of the reaction media was maintained slightly alkaline due to dissolved alkylamines. Purification of modified CNCs was conducted by washing in a water/iso-propanol (50/50 vol/vol) mixture which ensures the dissolution of alkylamines.

The presence of alkyl chains in modified CNCs was confirmed by ${ }^{1} \mathrm{H}-{ }^{13} \mathrm{C} \mathrm{CP} / \mathrm{MAS} \mathrm{NMR}$ experiments (Figure 1) To demonstrate that the alkyl moieties were covalently attached to CNCs, and not just physically adsorbed, we also carried out ${ }^{1} \mathrm{H}$ and ${ }^{1} \mathrm{H}^{-13} \mathrm{C} \mathrm{CP} / \mathrm{MAS}$ NMR experiments

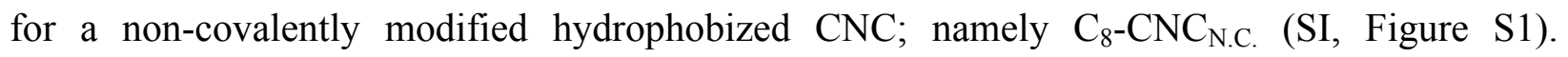
Interestingly, while the ${ }^{1} \mathrm{H}$ NMR spectrum of non-covalently modified $\mathrm{C}_{8}-\mathrm{CNC}_{\mathrm{N} . \mathrm{C}}$. clearly showed the presence of alkyl groups in the material, no alkyl peaks were detected in the ${ }^{1} \mathrm{H}-{ }^{13} \mathrm{C} \mathrm{CP} / \mathrm{MAS}$ experiment (10-50 ppm; SI, Figure S1). It is most likely that the alkyl chains in this material are too mobile to cross-polarize effectively, hence are not covalently attached to the CNC surface. The ${ }^{1} \mathrm{H}-{ }^{13} \mathrm{C} \mathrm{CP} / \mathrm{MAS}$ NMR can be used to probe for covalent surface functionalization of CNCs, and we have demonstrated that covalent hydrophobization was successful for $\mathrm{C}_{6^{-}}, \mathrm{C}_{8^{-}}$and $\mathrm{C}_{12}-\mathrm{CNC}$ samples (Figure 1).

The spectral deconvolution of the $i \mathrm{C} 6$ and $s \mathrm{C} 6$ peaks from ${ }^{1} \mathrm{H}^{-13} \mathrm{C} \mathrm{CP} / \mathrm{MAS}$ NMR spectra enabled us to estimate the surface areas (Eq. S1, S2) of non-modified $\mathrm{CNC}, \mathrm{C}_{6}-\mathrm{CNC}_{\text {and }} \mathrm{C}_{12}-\mathrm{CNC}$ (Table 1; SI, Figures S2, S3). Similar surface areas were obtained for $\mathrm{C}_{6}-\mathrm{CNC}$ and $\mathrm{C}_{12}-\mathrm{CNC}$, which are comparable to the value previously reported for $\mathrm{C}_{8}-\mathrm{CNC}^{48}$ (within the experimental error, Table 1). In contrast, non-modified CNC showed a surface area significantly larger than the three hydrophobized CNCs (SI, Figures S2). From the same ${ }^{1} \mathrm{H}^{-13} \mathrm{C} \mathrm{CP} / \mathrm{MAS}$ NMR, the spectral deconvolution of the alkyl peaks of $\mathrm{C}_{6}-\mathrm{CNC}$ and $\mathrm{C}_{12}-\mathrm{CNC}$ (SI, Figure S3) was used to calculate the degree of surface functionalization (DSF, Eq. S3) of modified CNCs. A DSF of $3.6 \pm 0.4$ and 
$2.6 \pm 0.3 \%$ was determined for $\mathrm{C}_{6}-\mathrm{CNC}$ and $\mathrm{C}_{12}-\mathrm{CNC}$, respectively (Table 1 ). Hence, the DSF of $\mathrm{C}_{6}$-CNC is comparable, within the experimental error, to the value reported previously for $\mathrm{C}_{8^{-}}$ $\mathrm{CNC}^{48}$, whereas degree of functionalization was slightly less efficient for $\mathrm{C}_{12}$-CNC. It might be caused by lower solubility of dodecylamine compared with hexyl- and octylamine.

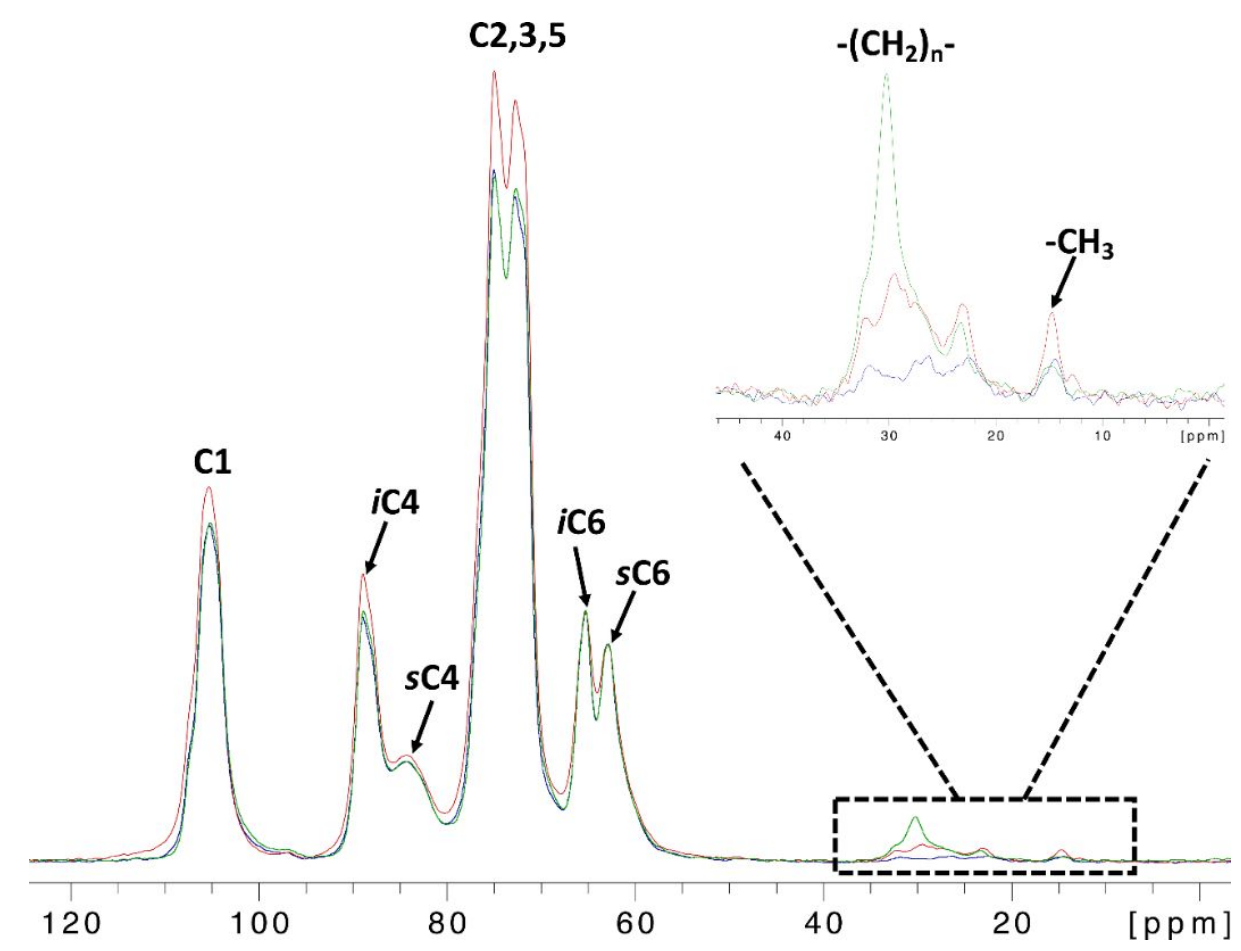

Figure 1. A typical ${ }^{1} \mathrm{H}_{-}{ }^{13} \mathrm{C} \mathrm{CP} / \mathrm{MAS}$ NMR spectra of covalently hydrophobized $\mathrm{C}_{6}$-CNC (blue), $\mathrm{C}_{8}-\mathrm{CNC}\left(\right.$ red, from ${ }^{48}$ ) and $\mathrm{C}_{12}-\mathrm{CNC}$ (green) powders acquired at $12 \mathrm{kHz}$ MAS rate at room temperature. The ${ }^{13} \mathrm{C}$ peaks corresponding to the alkyl moieties are magnified in the inset. The three spectra are scaled to match the same intensity for the $s \mathrm{C} 6$ peak. 
Table 1. Summary of the calculated parameters obtained from spectral deconvolution of iC6 and sC6 peaks of the ${ }^{1} \mathrm{H}^{13} \mathrm{C} \mathrm{CP}$ spectra of covalently hydrophobized $\mathrm{C}_{6}-\mathrm{CNC}, \mathrm{C}_{8}-\mathrm{CNC}$ and $\mathrm{C}_{12}-\mathrm{CNC}$ powders.

\begin{tabular}{|l|l|l|l|l|}
\hline & CNC & $\mathbf{C}_{6}$-CNC & $\mathbf{C}_{8}$-CNC* & $\mathbf{C}_{\text {12 }}$-CNC \\
\hline $\mathrm{q}$ & $0.57 \pm 0.02$ & $0.49 \pm 0.02$ & $0.52 \pm 0.02$ & $0.51 \pm 0.02$ \\
\hline$\sigma_{\text {fibril }}\left[\mathrm{m}^{2} \cdot \mathrm{g}^{-1}\right]$ & $805 \pm 40$ & $674 \pm 34$ & $693 \pm 35$ & $701 \pm 35$ \\
\hline DSF [\%] & $\mathrm{n} / \mathrm{a}$ & $3.6 \pm 0.4$ & $4.1 \pm 0.4$ & $2.6 \pm 0.3$ \\
\hline
\end{tabular}

* From Nigmatullin et al. ${ }^{48}$

\section{Physicochemical and surface properties of hydrophobized CNCs}

The CNCs used in this study were produced by hydrolysis in concentrated sulfuric acid solution. It is well-known that such an isolation of CNCs results in highly charged rod-shaped nanoparticles due to esterification of cellulose hydroxyl groups with sulfuric acid ${ }^{51,53}$. The $-\mathrm{SO}_{3} \mathrm{H}^{-}$group content for CNCs used in this study was around $235 \mathrm{mmol} \mathrm{kg}^{-1}$ of $\mathrm{CNC}$ determined from conductometric titration. This results in nanoparticles with high negative surface charge as characterized by zeta-potential, ca. $-48 \mathrm{mV}$ (Table 2), which plays a crucial role in their colloidal stability. ${ }^{54,55}$. Hydrophobization of the CNCs via binding of alkylamines was accompanied by a decrease of the sulfate half-ester group content by almost half (Table 2). Nevertheless, the zeta-potential, the determination of which is based on the electrophoretic mobility of the nanoparticles, decreased by only few units for hydrophobized CNCs. Only small variations in sulfate group content and zeta-potentials were observed for CNCs modified with alkylamines of different lengths. 
Sulfate half-esters in cellulose derivatives give several characteristic Raman bands (Zhang, Brendler et al. 2010). The Raman bands located at $\sim 825 \mathrm{~cm}^{-1}$ and $\sim 1270 \mathrm{~cm}^{-1}$, attributed to the $\mathrm{C}-$ $\mathrm{O}-\mathrm{S}$ stretching vibration and the $\mathrm{O}=\mathrm{S}=\mathrm{O}$ asymmetric stretching vibrations respectively, decreased after CNCs oxidation with sodium periodate (SI, Figure S4). This confirms that partial desulfation of CNCs took place during the oxidation stage. Despite this desulfation, a significant fraction of sulfate half-ester groups remained intact in hydrophobized CNCs. The absolute value of zeta-potentials of modified CNCs did not decrease below $\sim 40 \mathrm{mV}$ which is usually considered to be sufficient for high nanoparticle colloidal stability in aqueous suspensions.

Table 2. Surface properties of initial and hydrophobized CNCs

\begin{tabular}{|l|l|l|l|}
\hline CNC Type & $-\mathrm{SO}_{3} \mathrm{H}^{-}, \mathrm{mmol} \mathrm{kg}^{-1}$ & Zeta potential, $\mathrm{mV}$ & $\begin{array}{l}\text { Water contact } \\
\text { angle, }\end{array}$ \\
\hline $\mathrm{CNCs}$ & $235 \pm 30$ & $-48.3 \pm 0.5$ & $40.6 \pm 2.5$ \\
\hline $\mathrm{C}_{6}-\mathrm{CNCs}$ & $112 \pm 18$ & $-41.4 \pm 1.2$ & $59.5 \pm 1.3$ \\
\hline $\mathrm{C}_{8}-\mathrm{CNCs}$ & $118 \pm 8$ & $-44.1 \pm 0.8$ & $62.6 \pm 2.6$ \\
\hline $\mathrm{C}_{12}-\mathrm{CNCs}$ & $131 \pm 13$ & $-43.3 \pm 0.6$ & $66.0 \pm 0.5$ \\
\hline
\end{tabular}

To confirm the introduction of hydrophobic domains to the surface, water contact angles were measured for $\mathrm{CNC}$ films prepared by drying $1 \mathrm{wt} . \%$ suspensions on glass slides. The water contact angle for the unmodified $\mathrm{CNC}$ film was $\sim 40^{\circ}$ demonstrating wettability with water and thereby CNC hydrophilicity (Table 1 and SI, Figure S5). However, wettability of films made of modified CNCs notably decreased and water contact angles rose to $>60^{\circ}$. A gradual increase in water contact angle was observed with an increase in alkylamine chain length. Thus, modified CNCs are more 
hydrophobic than CNCs, and hydrophobicity of modified CNCs increased in the following order: $\mathrm{C}_{6}-\mathrm{CNCs}<\mathrm{C}_{8}-\mathrm{CNCs}<\mathrm{C}_{12}-\mathrm{CNCs}$.

Binding hydrophobic groups to the $\mathrm{CNC}$ surface is expected to change the microenvironment in aqueous $\mathrm{CNC}$ dispersions, similar to surfactant layers adsorbed onto particle surfaces. ${ }^{56}$ Pyrene, the fluorescence emission of which is sensitive to the solvent polarity, is a common probe in micellar systems and has been used to characterize microenvironments at interfaces. ${ }^{57}$ Figure 2 presents the changes in the intensity ratios for the third $\left(I_{3}\right)$ and first $\left(I_{1}\right)$ vibrionic bands of pyrene fluorescent emission at 382 and $370 \mathrm{~nm}$ respectively with concentration for different modified CNCs. Hydrophilic unmodified CNCs had very little effect on the pyrene fluorescence response, with $I_{3} / I_{1}$ ratios not exceeding 0.56 , a value characteristic for water. Similar values were observed for modified CNCs when their concentrations were below $0.25 \mathrm{wt} . \%$. Thus, under these conditions, pyrene was in a polar, hydrophilic microenvironment. In contrast, $I_{3} / I_{1}$ values for modified CNCs monotonically increased with concentration providing evidence that the microenvironment became increasingly non-polar in aqueous dispersions of modified CNCs. In a similar trend to contact angle measurements, $\mathrm{C}_{12}$-CNCs generated the most non-polar microenvironment followed by $\mathrm{C}_{8}$ - and $\mathrm{C}_{6}$-CNCs. $I_{3} / I_{1}$ values for modified $\mathrm{CNCs}$ ranged from 0.51 to 0.94 , which are typical values for aqueous micellar systems. ${ }^{56,58}$ However, the concentration dependence of $I_{3} / I_{1}$ ratios in surfactant systems has an S-shaped pattern with a sharp increase around the critical micellar concentration due to the increase of pyrene solubilization in micelles. The observed monotonic increase in $I_{3} / I_{1}$ ratio with increasing modified $\mathrm{CNC}$ concentration is probably due to the combination of two different mechanisms of pyrene solubilization: solubilization at interfaces of CNCs containing hydrophobic domains and solubilization in a volume formed via aggregation 
driven by hydrophobic effects. The latter is similar to pyrene solubilization in micelles, whilst the former has been reported for dispersion of hydrophobized particles or particles with adsorbed surfactants. 56,57

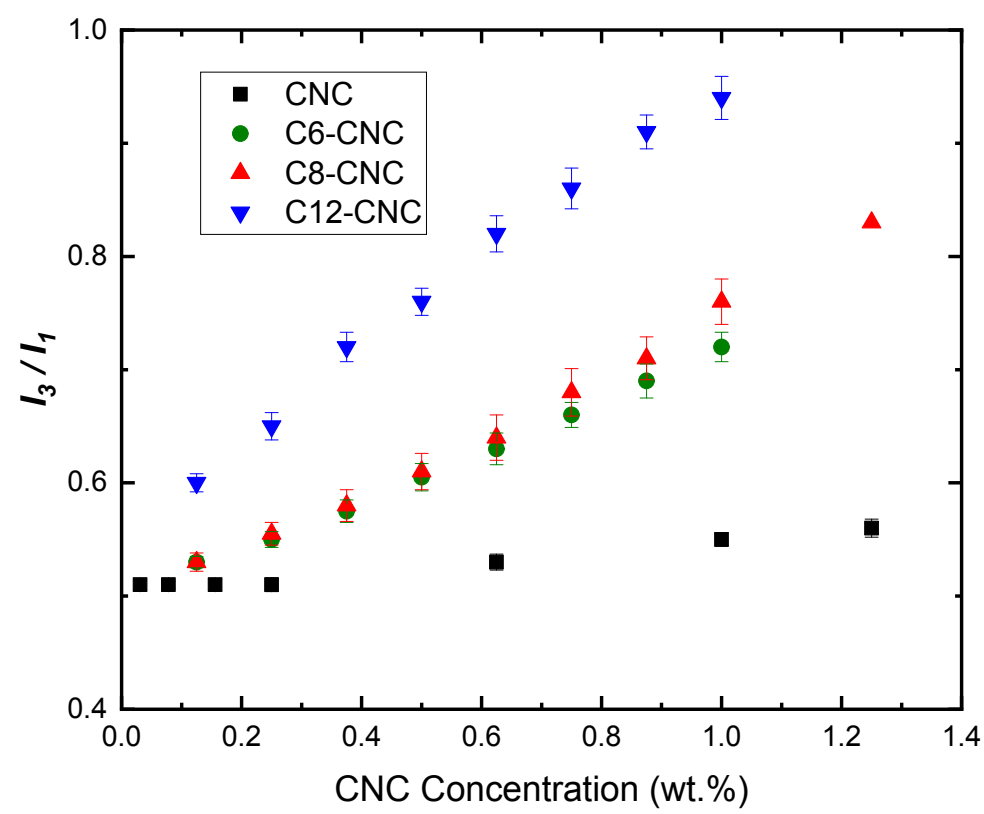

Figure 2. Change in the pyrene fluorescent emission intensity ratio $I_{3} / I_{1}$ in aqueous suspensions of unmodified $(\mathrm{CNC})$ and modified $\mathrm{CNCs}\left(\mathrm{C}_{6}-\mathrm{CNC}, \mathrm{C}_{8}-\mathrm{CNC}, \mathrm{C}_{12}-\mathrm{CNC}\right)$ with a variation in the concentration of CNCs.

Characterization of surface properties of modified CNCs provides evidence that both hydrophilic and hydrophobic domains are present on the surface of modified CNCs. As a result, modified CNCs exhibited surface activity and decrease interfacial tension (Figure 3). Unmodified CNCs did not however induce a decrease in the interfacial tension in suspensions with concentrations up to $0.8 \mathrm{wt} \%$ (data not shown). However, there appeared to be no direct correlation between the length of alkyl chains attached to the CNC surface and surface activity. The highest decrease in surface tension, to $\sim 51 \mathrm{mN} \mathrm{m}^{-1}$, was observed for the $\mathrm{C}_{8}$-CNCs suspension 
at concentrations higher than 0.25 wt.\%. Despite showing higher hydrophobicity, demonstrated by in contact angle and pyrene probe measurements, $\mathrm{C}_{12}-\mathrm{CNCs}$ caused only a moderate decrease (to $\sim 62 \mathrm{mN} \mathrm{m}^{-1}$ ) in surface tension. Stronger hydrophobic effects in $\mathrm{C}_{12}$-CNCs suspensions might induce association of the $\mathrm{C}_{12}-\mathrm{CNCs}$ and decrease the content of individualized CNCs in the suspension. Since individualized CNCs are expected to be drawn to the interface, $\mathrm{C}_{12}$-CNCs had lower surface activity in comparison with $\mathrm{C}_{8}$-CNCs. The lowest surface activity was exhibited by $\mathrm{C}_{6}$-CNCs, suggesting that the surface charge and other hydrophilic cellulose groups prevail over the hydrophobic domains, resulting in their dispersion in the water bulk rather than being drawn to the air/water interface. All three hydrophobized CNCs showed concentration dependence typical for amphiphiles, exhibiting concentration dependent segments at low concentrations, and regions of constant surface tension when concentration exceeded a critical value. A critical aggregation concentration (CAC), defined as the onset of a steady state value of surface tension, is associated with the formation of aggregated structures in the bulk of the solution. The CAC values for $\mathrm{C}_{6^{-}}, \mathrm{C}_{8^{-}}$, and $\mathrm{C}_{12}$-CNCs were found to be ca. $0.51,0.25$, and $0.27 \mathrm{wt} \%$, respectively (Table 2). There were no detectable changes in the form of the pyrene emission curves (Figure 2) around these $\mathrm{CAC}$ values, confirming the hypothesis that different mechanisms are involved in pyrene solubilization in these systems. This is unlike for micellar systems where pyrene is mostly solubilized in micelles with a drastic increase in the $I_{3} / I_{1}$ ratio at concentrations close to the critical micellar concentration. 


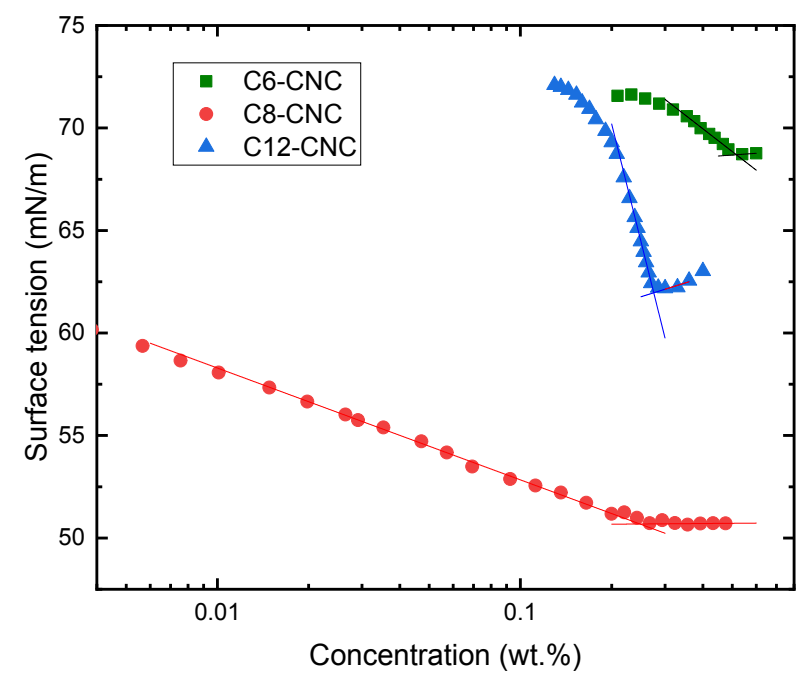

Figure 3. Dependence of modified CNC surface tension on concentration. Solid lines are linear fits of a plateau region and linearly dependent regions preceding the plateau. Critical aggregation concentrations (CAC) ca. 0.51, 0.25, and 0.27 wt.\% were determined for $\mathrm{C}_{6^{-}}, \mathrm{C}_{8^{-}}$, and $\mathrm{C}_{12^{-}} \mathrm{CNCs}$ respectively as the intersection between these linear fits.

\section{Structure of aqueous CNC suspensions}

To probe the effect of hydrophobization on CNC interaction in aqueous media, multi-channel confocal laser scanning spectroscopy (MCLSS) and SAXS experiments were conducted for unmodified and modified CNC suspensions at various concentrations. The former technique is based on the autofluorescence of cellulose materials. Recently it was demonstrated that the autofluorescence of cellulose could be used to track microfibrils and nanocrystals in composite structures. ${ }^{44,59}$ Two autofluorescent emission bands were found to be dominant; one at 463.5-472.5 $\mathrm{nm}$ (herein referred to as the $468 \mathrm{~nm}$ band) and one at $499.5-508.5 \mathrm{~nm}$ (herein referred to as the $504 \mathrm{~nm}$ band). We theorize that the $468 \mathrm{~nm}$ band is related to intra-particle forces (hydrogen 
bonding, van der Waals forces and electrostatic forces), whilst the $504 \mathrm{~nm}$ band is related to those associated with inter-particle interactions due to an expected lower excitation-emission conversion efficiency. To confirm this, the ratio between the 468 and $504 \mathrm{~nm}$ bands was tracked as the concentration of CNCs was increased from 0.1 to 11 wt.\% (Figure 4 and SI, Figure S6). As the concentration increases, the ratio between the two bands decreases, suggesting an increase in the inter-crystal interactions. Taking the initial and final gradients of an exponential curve according to the equation

$$
y=A e^{(1-B x)}+C
$$

fitted to the data, where A, B and C are constants (see Table 2 for fit data), an interception point for unmodified CNCs is 3.28 wt.\% (Table 2), which falls within the range at which chiral nematic phases are known to form for CNC suspensions ${ }^{60,61}$; this confirms the fluorescent ratio dependence on $\mathrm{CNC}$ interaction. Hydrophobization of the CNCs results in their aggregation, as inferred from the interception point, at lower concentrations than the hydrophilic CNCs (Table 2) with the chain length series $\left(\mathrm{C}_{8}<\mathrm{C}_{12}<\mathrm{C}_{6}\right)$ matching that observed in surface tension experiments.

Further information on the nature of the interactions between the hydrophobic CNCs may also be obtained from the absolute 468:504 nm emission ratio values at theoretical concentrations of 0 and 100 wt.\%. Unlike the other materials, the $\mathrm{C}_{6}$-CNCs exhibit an increase in the emission ratio as the concentration increases. We theorize that the secondary amine groups present, formed as a result of alkylamine coupling, interacts with the sulfate half-ester groups present on the CNC surface. This results in a hypsochromic (blue) shift in the spectrum and an increase in the emission intensity 
(SI, Figure S6, Figure S7). We confirm that this is feasible by observing a similar shift when a CNC gel is combined with a chitosan solution (SI, Figure S8). In contrast, the length of the C8 and C12 carbon chains inhibit this interaction, resulting in the typical increase in the $504 \mathrm{~nm}$ band upon increasing concentration.

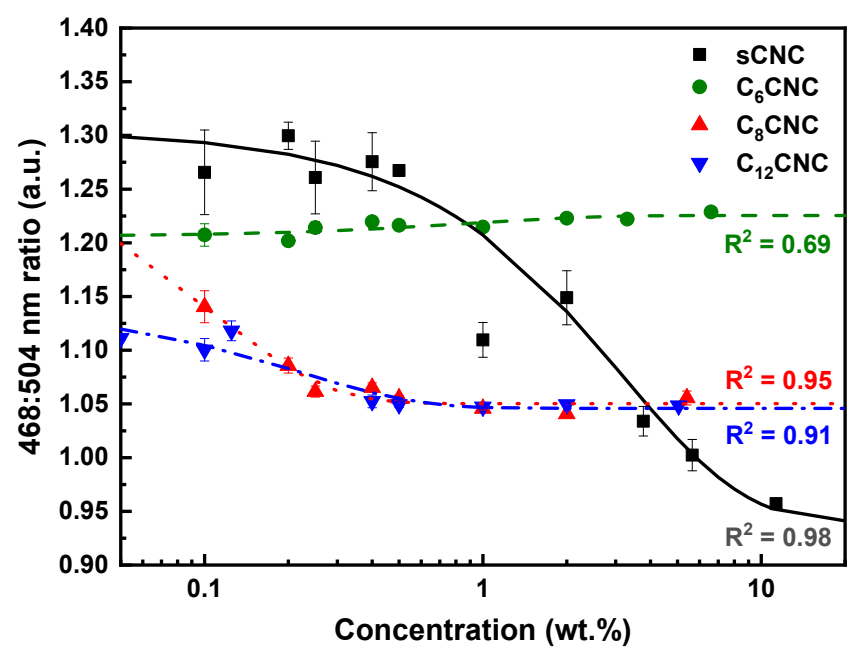

Figure 4. Effect of CNM concentration on ratio of emission intensity at $468 \mathrm{~nm}$ (associated with intra-crystal fluorescence) and $504 \mathrm{~nm}$ (associated with inter-crystal fluorescence). Exponential curves (Eqn. 1) fitted for each data series. Error bars: \pm S.E. $N=1, n=3$. 
Table 2. Select data for CNC and modified CNC suspensions as determined by surface tension measurements and exponential curves fitted to MCLSS data.

\begin{tabular}{|c|c|c|c|c|c|}
\hline \multicolumn{2}{|l|}{ CNM } & CNCs & $\mathrm{C}_{6}-\mathrm{CNCs}$ & $\mathrm{C}_{8}$-CNCs & $\mathrm{C}_{12}$-CNCs \\
\hline \multicolumn{2}{|l|}{ CAC [wt.\%] } & - & 0.51 & 0.25 & 0.27 \\
\hline \multicolumn{2}{|c|}{$\begin{array}{l}\text { MCLSS } \\
\text { interception point [wt.\%] }\end{array}$} & $3.28 \pm 0.09$ & $1.40 \pm 0.77$ & $0.16 \pm 0.00$ & $0.25 \pm 0.01$ \\
\hline \multirow{2}{*}{$\begin{array}{l}468: 504 \mathrm{~nm} \\
\text { ratio [a.u.] }\end{array}$} & 0 wt. $\%$ & $1.30 \pm 0.01$ & $1.20 \pm 0.01$ & $1.30 \pm 0.02$ & $1.14 \pm 0.00$ \\
\hline & 100 wt. $\%$ & $0.94 \pm 0.00$ & $1.23 \pm 0.00$ & $1.05 \pm 0.00$ & $1.04 \pm 0.00$ \\
\hline
\end{tabular}

From SAXS experiments (Figure 5 and SI, Figures S9 and S10), unmodified CNC rods (Figure 5a) are found to have an elliptical cross-section $\left(R_{\min }=1.6 \pm 0.1\right.$ and $\left.R_{\max }=15.0 \pm 0.1 \mathrm{~nm}\right)$ and a length $L$ fixed at $\sim 110 \mathrm{~nm}$ in agreement with TEM measurements. With increasing concentration, a growing correlation peak emerges. This peak sharpens with concentration and moves towards larger $q$ values (around $\mathrm{q} \sim 0.015 \AA^{-1}$ at $10 \mathrm{wt} . \%$ ). This correlation peak is due to increased excluded volume interactions between unmodified CNCs and is modelled using the PRISM model. From the fits, two parameters are extracted: excluded volume parameter, $v_{\mathrm{RPA}}$, which is dependent on the interacting rod concentration, and the radius of excluded volume, $R_{\mathrm{cq}}$, associated with the inter cylinder distance in concentrated regimes (Figure 6). $v_{\mathrm{RPA}}$ was found to increase linearly with concentration, as is expected from increasing particle-particle interactions, due to the electrostatic repulsion between the charged nanorods. On the other hand, the local excluded volume parameter $R_{\text {cq }}$ decreased with concentration, indicating a denser packing of CNCs. The decrease is pronounced until a 4 wt.\% CNC concentration, and much weaker for concentrations above this value. Moreover, the fits show differences with the data in the small $q$ range (see individual fits in 
SI, Figure S9). This is probably due to the fact that the isotropic-to-nematic phase transition is reached at concentration above 4 wt.\%, and the suspensions are biphasic with liquid-crystalline and isotropic regions. ${ }^{60}$ This will be further discussed in the rheology section.
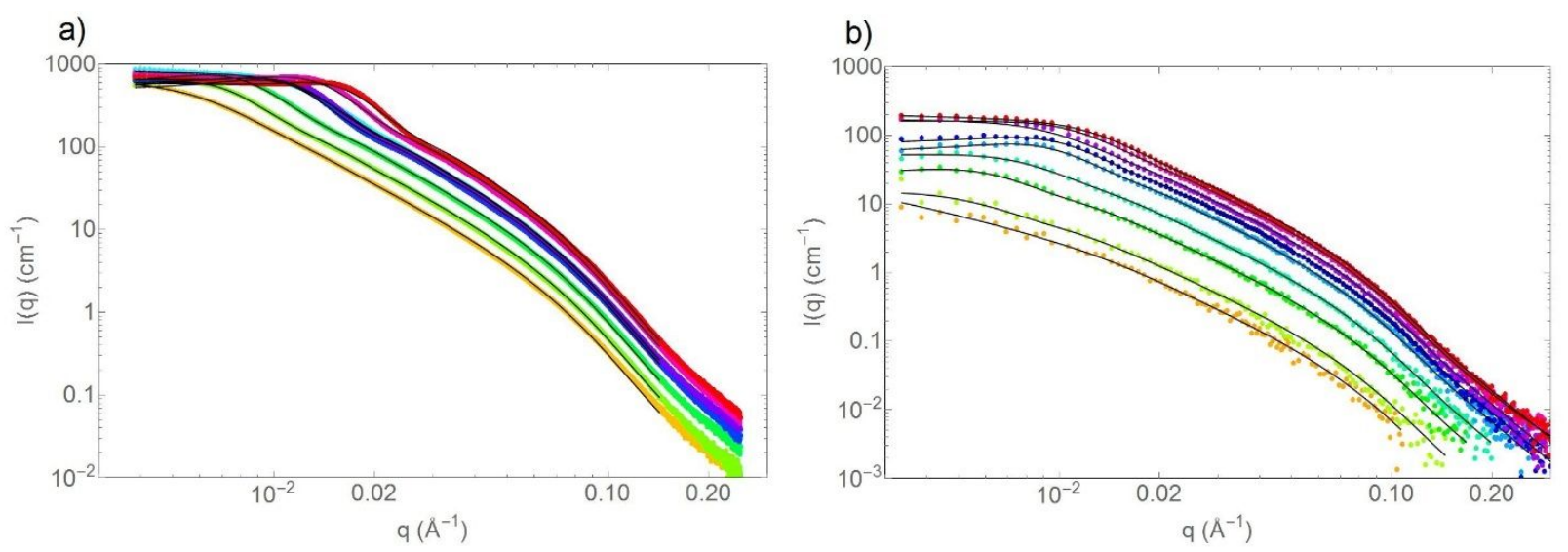

Figure 5: SAXS patterns, $I(q)$ versus $q$, in absolute scaling for (a) unmodified CNC suspensions (from Diamond) and (b) $\mathrm{C}_{8}$-CNC (Ganesha) at various concentrations. The fits made using the model of rigid interacting cylinders are given as solid black lines. The same patterns are plotted individually in Figure S9 and S10 in SI. Data measured at Diamond for $\mathrm{C}_{8}-\mathrm{CNC}$ were also recorded for low concentrations and are given in Figure S11. 

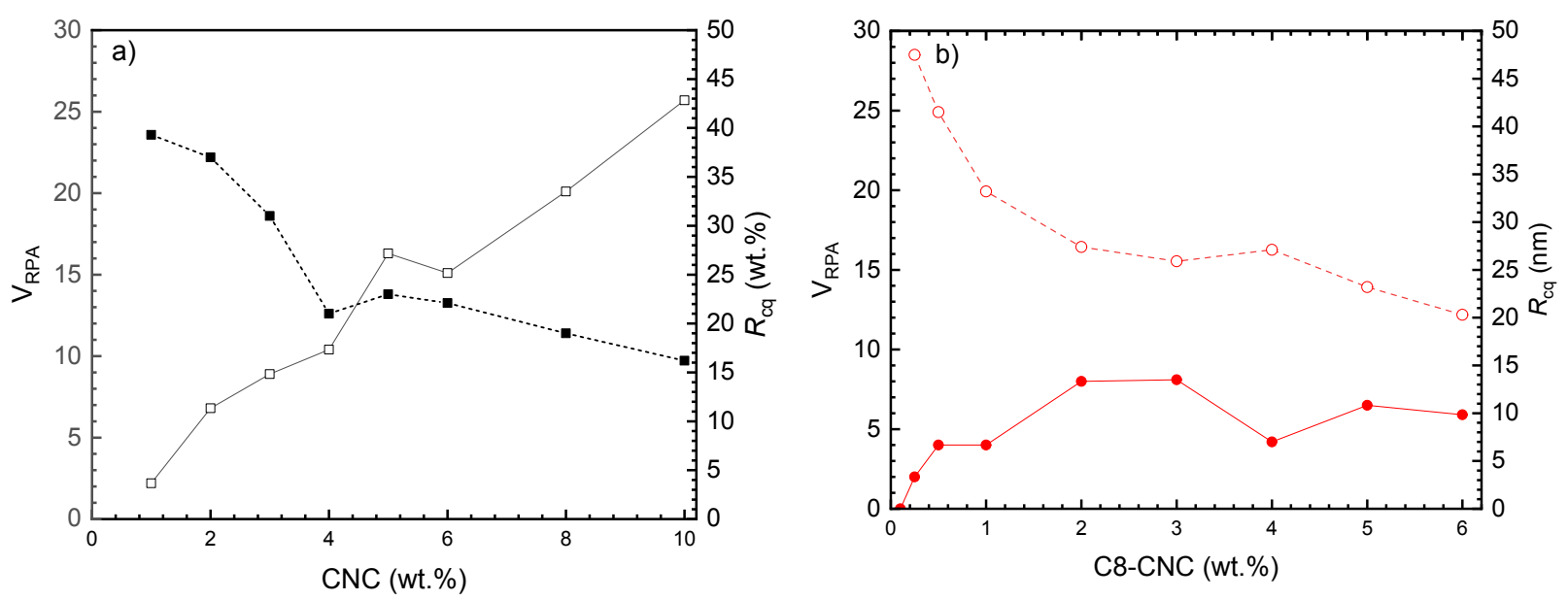

Figure 6: The influence of CNC concentration on $v_{\mathrm{RPA}}$ (filled symbols) and $R_{\mathrm{cq}}$ (open symbols) determined from SAXS experiments for unmodified CNCs (a) and $\mathrm{C}_{8}-\mathrm{CNC}$ (b). The lines are a guide to the eye.

To study the effect of CNC surface modification, $\mathrm{C}_{8}$-CNCs suspension were also characterized by SAXS at various concentrations ( 0.1 to $6 \mathrm{wt} . \%)$ (Figure $5 \mathrm{~b})$. From the fit in the dilute regime (0.1 wt.\%), where the signal is attributed to isolated CNCs with negligible interactions, the form factor of the particles can be extracted. The cross-section of the rods is found to be $R_{\min }=1.6 \pm 0.1$ $\mathrm{nm}$ and $R_{\max }=9.2 \pm 0.1 \mathrm{~nm}$ for the minor and major radii respectively. Modification of the CNC induced a significant reduction in the major radius compared to unmodified CNCs. Above 0.25 wt.\%, interactions between $\mathrm{C}_{8}$-CNCs are needed to fit the data. Interestingly, in opposition to unmodified $\mathrm{CNC}, v_{\mathrm{RPA}}$ increases only up to ca. 2 wt.\% (with slightly stronger repulsion for $\mathrm{C}_{8}$-CNC than their unmodified counterparts at 1 and $2 \mathrm{wt} \%$ ). Above 2 wt.\%, $v_{R P A}$ remains relatively constant. A similar trend is observed for $R_{\mathrm{cq}}$, which strongly decreases up to $2 \mathrm{wt} . \%$, before stabilizing, or only weakly decreasing, to reach $20 \mathrm{~nm}$ at $6 \mathrm{wt} . \%$. The rapid stabilization of the repulsion forces in the system around ca. $2 \mathrm{wt} . \%$ corresponds to the concentration at which an 
invertible gel is formed with $\mathrm{C}_{8}$-CNC (SI, Figure S12). Hence, this trend could be explained by the formation of the self-standing gel, with a network spanning throughout the entire suspension, with contact points between CNCs at larger dimensions than probed in this q-range formed between $\mathrm{C}_{8}$-CNC nanorods due to hydrophobic effects. This would "freeze" the $\mathrm{C}_{8}$ - $\mathrm{CNC}$ in a disordered fashion, preventing the formation of a nematic phase.

\section{Rheological properties of CNC suspensions}

The rheological properties of aqueous suspensions of CNCs produced by hydrolysis with sulfuric acid have been previously characterized in detail. ${ }^{54,60-63}$ These previously published works also covered suspensions of CNCs of the same source as used in our study. ${ }^{63}$ It is generally agreed that the rheological properties of suspensions of unmodified CNCs are defined by their ability to form biphasic systems of isotropic and chiral nematic phases. CNC suspensions exhibit Newtonian fluid behavior at low concentrations when suspensions are isotropic. However, at higher concentrations $\mathrm{CNCs}$ form a liquid crystalline phase transforming the suspension into biphasic system with the liquid crystal phase fraction being dependent on $\mathrm{CNC}$ concentration. These structural changes transform the suspensions into viscoelastic fluids. Ultimately, at sufficiently high concentrations, randomly entangled gels are formed. The aspect ratio and surface charge of the rod-like cellulose nanoparticles, and the ionic strength of the aqueous media determine the critical concentrations for the transitions between these states.

The rheological properties of hydrophobized and unmodified CNCs were investigated at a wide range of concentrations, which covered systems from fluids to gels. Our results for unmodified $\mathrm{CNCs}$ are in good agreement with previous findings ${ }^{54,60-63}$ as outlined above. For example, 
frequency sweeps in oscillatory rheology demonstrated that suspensions of unmodified CNCs exhibited liquid-like behavior $\left(\mathrm{G}^{\prime \prime}>\mathrm{G}^{\prime}\right)$ for $\mathrm{CNC}$ concentrations $<5$ wt.\% (SI, Figure S13a). When concentrations exceeded $5 \mathrm{wt}$ \% elastic properties become dominant $\left(\mathrm{G}^{\prime}>\mathrm{G}^{\prime \prime}\right)$. Hydrophobization of CNCs drastically changed the viscoelastic properties, significantly increasing values of G' (SI, Figure S13 b-d). Dominance of elastic properties $\left(\mathrm{G}^{\prime}>\mathrm{G}^{\prime \prime}\right)$ was extended to the suspensions with concentrations as low as $1 \mathrm{wt} . \%$ for $\mathrm{C}_{8}-\mathrm{CNCs}$ and $\mathrm{C}_{12}-\mathrm{CNCs}$ but only to $4 \mathrm{wt} . \%$ for $\mathrm{C}_{6}-\mathrm{CNCs}$. Interestingly, values of $\mathrm{G}^{\prime}$ were larger than $\mathrm{G}^{\prime \prime}$ for suspensions of hydrophobized CNCs that did not form invertible gels (concentrations forming invertible gels were 1.5, 2.5 and $4 \mathrm{wt} \%$ for $\mathrm{C}_{12^{-}}$, $\mathrm{C}_{8^{-}}$and $\mathrm{C}_{6^{-}} \mathrm{CNCs}$ respectively, SI, Figure $\mathrm{S} 12$ ). For suspensions at concentrations below the invertible gel concentration, differences in $\mathrm{G}^{\prime}$ and $\mathrm{G}^{\prime \prime}$ were less than a decade $(\tan \delta \approx 0.1)$. These values are characteristic for "weak gels" or "structured fluids" which are usually formed by tenuous association of mesoscopic domains. ${ }^{64}$

The frequency dependence of the storage modulus was analyzed quantitatively by fitting a simple power law relationship, according to the equation

$$
G^{\prime} \propto \omega^{p}
$$

where $\omega$ is the angular frequency of oscillation and $p$ is the storage modulus power law index.

There were only slight variations in the power law index in the studied range of concentrations for $\mathrm{C}_{8}$ - $\mathrm{CNCs}$ and $\mathrm{C}_{12}$-CNCs. For $\mathrm{C}_{8}$-CNCs, $p$ decreased slightly from 0.09 for a $1 \mathrm{wt} . \% \mathrm{C}_{8}$-CNC suspension to 0.07 for $7 \mathrm{wt} . \%$, whilst $p$ was 0.06 for $1 \mathrm{wt} . \% \mathrm{C}_{12}-\mathrm{CNCs}$ and was constant (ca. 0.04) for concentrations between 2 to 5 wt.\%. Thus, the frequency independence of $\mathrm{G}^{\prime}$ was observed 
even for suspensions at the lowest concentrations of $\mathrm{C}_{8}-\mathrm{CNCs}$ and $\mathrm{C}_{12}$-CNCs. However, for $\mathrm{C}_{6}$-CNCs, $\mathrm{G}^{\prime}$ was strongly dependent on frequency for the suspensions with concentrations between 2 and 4 wt.\% ( $p$ was ca. 2, 0.7 and 0.12 for 2, 3, and 4 wt.\%, respectively). A frequency independent $\mathrm{G}^{\prime}$ was only observed for $\mathrm{C}_{6}-\mathrm{CNC}$ suspensions when concentration exceeded 5 wt.\%.

The viscoelastic properties of unmodified and hydrophobized CNCs were compared at different CNC concentrations (Figure 7). As expected, $\mathrm{G}^{\prime}$ increased with concentration for all CNCs. However, rheological properties are strongly dependent on the hydrophobicity of CNCs, and suspensions and gels of $\mathrm{C}_{8^{-}}$and $\mathrm{C}_{12}$-CNCs exhibited significantly higher $\mathrm{G}^{\prime}$ values compared with suspensions of unmodified CNCs and $\mathrm{C}_{6}$-CNCs at the same concentration. For example, at 5 wt.\%, the $\mathrm{G}^{\prime}$ value of an $\mathrm{C}_{8}$-CNC gel suspension is higher than for unmodified CNCs by almost five orders of magnitude; with values of 4300 and 0.05 Pa respectively. $\mathrm{G}^{\prime}$ increased further to $\sim 9000$ Pa for 5 wt. $\%$ of $\mathrm{C}_{12}$-CNCs. Modification with the shorter chain hexylamine resulted in a moderate increase in $\mathrm{G}^{\prime}$ to $c a .150 \mathrm{~Pa}$ for 5 wt.\% $\mathrm{C}_{6}$-CNCs. Also, a strong dependence of $\tan \delta$ on concentration was observed for unmodified $\mathrm{CNC}$ and $\mathrm{C}_{6}-\mathrm{CNC}$ suspensions while tan $\delta$ was below 0.08 for the complete concentration range for $\mathrm{C}_{8^{-}}$and $\mathrm{C}_{12}$-CNCs (Figure $7 \mathrm{~b}$ ). The elastic properties of unmodified $\mathrm{CNC}$ and $\mathrm{C}_{6}$ - $\mathrm{CNC}$ suspensions only became significant when concentrations exceeded 5 and 3 wt.\% respectively. When the concentrations increased beyond these points $\tan \delta$ fell below 1.0 which is usually attributed to gel formation. However, self-supported gels (invertible gels) are not formed when $\tan \delta$ is equal to 1.0 at $\mathrm{CNC}$ concentrations $3 \mathrm{wt} . \%$ for $\mathrm{C}_{6}$ - $\mathrm{CNCs}$ and 5 wt.\% for unmodified CNCs (SI, Figure S12). 

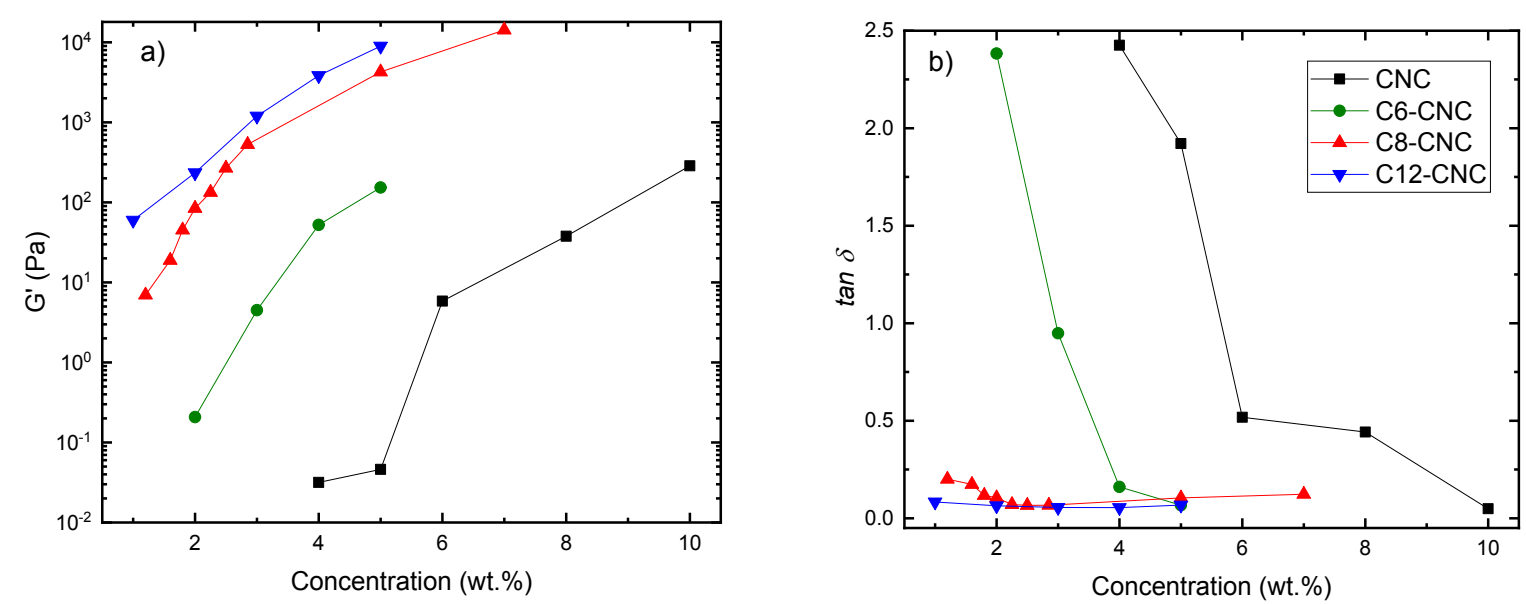

Figure 7. Storage modulus (a) and $\tan \delta(\mathrm{b})$ as a function of concentration of unmodified and hydrophobized CNCs at an angular frequency of $6.34 \mathrm{rad} \mathrm{s}^{-1}$ and strain $1.5 \%$.

It is well-known that the gel formation of $\mathrm{CNC}$ suspensions can be induced by the introduction of electrolytes. ${ }^{54,63,65}$ Such behavior has been observed for suspensions of charged particles other than CNCs and attributed to the aggregation of these particles with an increase in ionic strength. Viscoelastic properties of CNCs were hence also compared for suspensions prepared in $0.1 \mathrm{M} \mathrm{KCl}$ at a $\mathrm{CNC}$ concentration of $4 \mathrm{wt} \%$ (Figure 8). The presence of electrolyte induced a significant increase in $\mathrm{G}^{\prime}$ for unmodified $\mathrm{CNCs}$, and $\mathrm{G}^{\prime}$ became larger than $\mathrm{G}^{\prime \prime}$ ( $\tan \delta$ around 0.1 compared with 2.0 in the absence of salt). Suspensions of hydrophobized CNCs are characterized by higher $\mathrm{G}^{\prime}$ compared with unmodified CNCs. However, unlike the suspensions in DI water, there was no direct correlation with the number of carbon atoms of alkyl chain (hydrophobicity) and $\mathrm{G}^{\prime}$ values of suspensions with background electrolyte: $\mathrm{G}^{\prime}$ values for $\mathrm{C}_{12}-\mathrm{CNC}$ suspension were lower compared with values for the suspensions of $\mathrm{C}_{8}$-CNCs and $\mathrm{C}_{6}$-CNCs. In fact, $\mathrm{G}^{\prime}$ values for $\mathrm{C}_{12}$-CNCs suspensions in $0.1 \mathrm{KCl}$ were lower than in $\mathrm{DI}$ water whilst the background electrolyte induced an increase in $\mathrm{G}^{\prime}$ values for $\mathrm{C}_{6^{-}}$and $\mathrm{C}_{8}$ - $\mathrm{CNC}$ suspensions. It should be noted that 
hydrophobic effects in hydrophobized CNCs had more pronounced influence on $\mathrm{CNC}$ rheology compared with the electrolyte effect. For example, at an angular frequency $6.34 \mathrm{rad} \mathrm{s}^{-1}, \mathrm{G}^{\prime}$ of 5 wt.\% $\mathrm{C}_{8}$-CNC suspension in DI water was $4300 \mathrm{~Pa}$ in comparison with $880 \mathrm{~Pa}$ for 5 wt.\% suspension of unmodified $\mathrm{CNCs}$ in $0.1 \mathrm{M} \mathrm{KCl}$ solutions.

As was discussed in previous sections, surface functionalization of CNCs with hydrophobic moieties facilitate interactions between CNCs due to hydrophobic effects. Therefore, the formation of microgel aggregates is thought to occur even at relatively low concentrations of hydrophobized CNCs, especially for the more hydrophobic $\mathrm{C}_{8^{-}}$and $\mathrm{C}_{12}$-CNCs, leading to phase separation. The elastic behavior of such a weak gel is caused by the elasticity of the hydrophobized CNC microgel dispersed in the aqueous phase depleted of CNCs. However, such a system does not form self-supporting gels. With an increase in concentration of hydrophobized CNCs, a strong network of connected aggregates is formed, leading to strong gels. It is worth noting that electrolytes have strong effects on the rheological properties and gelation of hydrophobized CNCs. Therefore, structural and rheological properties of hydrophobized CNCs are defined by two competing phenomena: electrostatic repulsion and association driven by hydrophobic effects. Under different conditions ( $\mathrm{CNC}$ and electrolyte concentrations, hydrophobicity of modifying agent) one phenomenon can prevail over the other, for example, electrostatic repulsion is suppressed at higher ionic stress leading to the dominance of the hydrophobic effect. This is probably the reason for the decreased elastic properties of $\mathrm{C}_{12}$ - $\mathrm{CNC}$ gels in the presence of a background electrolyte (Figure 8). For these most hydrophobic CNCs, when electrostatic repulsion is suppressed the hydrophobic effect became too strong, inducing excessive $\mathrm{C}_{12}$-CNC aggregation. As a result, $\mathrm{G}^{\prime}$ is lower in the presence of electrolyte than in $\mathrm{C}_{12}-\mathrm{CNCs}$ hydrogel prepared in DI water. At the same time, $\mathrm{G}^{\prime}$ 
increased in the presence of $\mathrm{KCl}$ for $\mathrm{C}_{6}$ - and $\mathrm{C}_{8}$-CNCs. Gel formation was also observed at slightly higher concentrations of hydrophobized CNCs in $0.1 \mathrm{M} \mathrm{KCl}$ compared with suspensions in DI water (Data not shown). Thus, at relatively low concentrations of hydrophobized CNCs, the addition of electrolytes disrupted the network, causing the formation of a phase-separated system. These observations indicate that the surface charge contributes to the stabilization of the network formed via the $\mathrm{CNC}$ association driven by hydrophobic effects.

Rheological properties of CNC suspensions/gels were further characterized in steady-shear experiments (SI, Figure S14). The pattern of the response of unmodified CNC suspensions/gels to shear flow (SI, Figure S14a) was similar to previous independent studies. ${ }^{54,61,62}$ At CNC concentrations sufficiently high for forming biphasic systems, there are three regions in the flow curve (for example, curves for 5 and 6 wt. \% in Figure S14a). The low shear rate region presents a strong shear thinning behavior. It is followed by the region with a weak dependence of the viscosity on shear rate and another shear-thinning region at higher shear rates. The three-region pattern has been also previously reported for other liquid crystal systems. ${ }^{66,67}$ For CNCs it is generally agreed that the first shear thinning region is caused by alignment of the nematic liquid

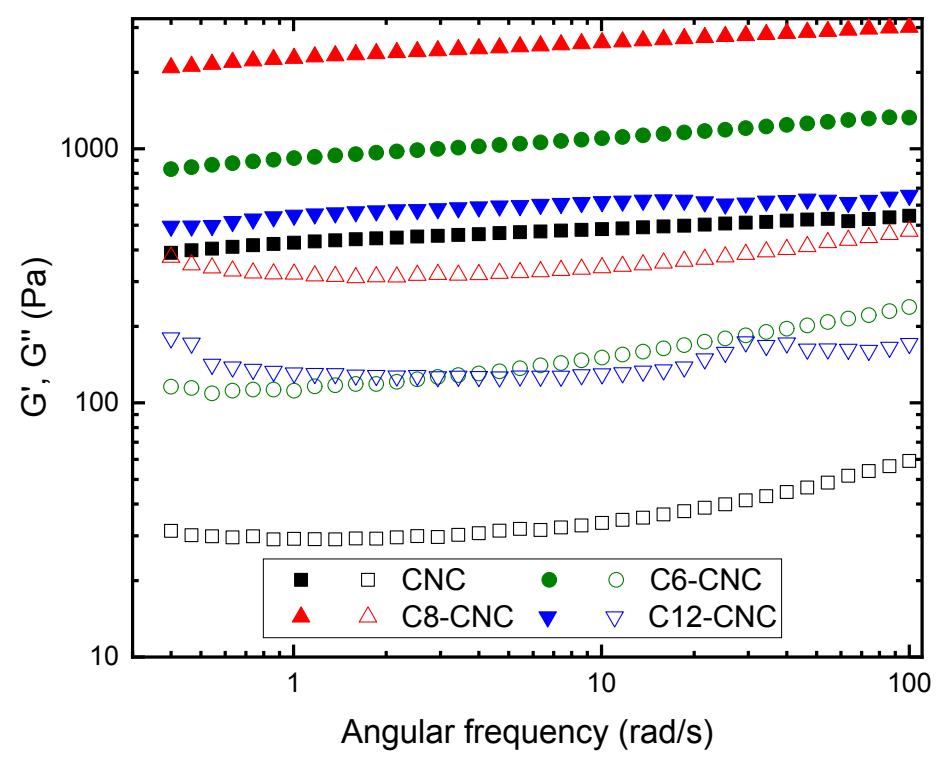


Figure 8. Dependence of storage modulus (filled symbols) and loss modulus (open symbols) on oscillation frequency of gels of unmodified and hydrophobized CNCs at 4 wt.\% in $0.1 \mathrm{M} \mathrm{KCl}$. Strain $1.5 \%$.

crystalline domains. When alignment of these domains is completed, an increase in shear rate destabilizes the nematic mesophase, which manifests as weak dependences of viscosity on shear rate. Finally, at high shear rates, shear thinning is due to alignment of the $\mathrm{CNC}$ nanorods. Different regions of viscosity sensitivity to the shear rate were observed at some concentrations only for $\mathrm{C}_{8^{-}}$ and $\mathrm{C}_{12}$-CNCs (SI, Figure S14 c, d). However, only one regime of shear thinning was observed for $\mathrm{C}_{6}$-CNCs suspension/gels in the studied range of concentrations. A set of viscosity values at various concentrations was extracted from flow curves for a shear rate of $0.1 \mathrm{~s}^{-1}$ and presented in Figure 9. In agreement with the results from oscillatory rheology experiments, viscosities of the gels based on hydrophobized CNCs are significantly higher than unmodified CNCs. For example, for systems containing 4 wt.\% of CNCs, shear viscosity increased from 0.07 Pa.s for unmodified CNCs to $c a .1350$ Pas in case of $\mathrm{C}_{12}$-CNCs. There was a direct correlation between hydrophobicity of CNCs (length of alkyl radical) and enhancement in viscosity (unmodified $\left.\mathrm{CNCs}<\mathrm{C}_{6}-\mathrm{CNCs}<\mathrm{C}_{8}-\mathrm{CNCs}<\mathrm{C}_{12}-\mathrm{CNCs}\right)$.

Dependence of the viscosity on concentration has been proposed as means for the identification of the transition of aqueous CNC systems from isotropic to biphasic. ${ }^{60}$ The region of a sharp increase of viscosity indicates transformation of the system into biphasic system. For CNCs used in this study this occurred at concentration around 5 wt.\% (Figure 9). Thus, quite high concentrations of $\mathrm{CNCs}$ used in this study are required to enable assembly into structured liquid 
crystalline domains. The Figure 9 also shows concentrations corresponding the formation of invertible gels. These concentrations for hydrophobized $\mathrm{CNCs}\left(1.5,2.5\right.$, and 4 wt.\% for $\mathrm{C}_{12^{-}}, \mathrm{C}_{8^{-}}$ and $\mathrm{C}_{6}-\mathrm{CNCs}$ respectively) are lower than the concentration of the transition into biphasic phase for unmodified CNCs. This might impede the formation of liquid crystalline phases in the suspensions of hydrophobized CNCs, which agrees with the $\mathrm{C}_{8}-\mathrm{CNC}$ SAXS data (no obvious variation of the interactions for concentration above the formation of an invertible gel). To check the formation of liquid crystalline phase microscopic images in cross-polarized light of the suspensions were obtained (SI, Figure S15). Formation of liquid crystalline domains was confirmed for unmodified CNCs. However, all hydrophobized CNCs did not exhibit birefringence, confirming no formation of a liquid crystalline phase at concentrations below, and above gel formation, again in agreement with the weak repulsive interaction observed in SAXS. Random association of hydrophobized CNCs arresting the CNC mobility in the gel state prevents the formation of a liquid crystalline phase. Earlier inhibition of the formation of ordered liquid crystalline structures due to gelation was assumed for the systems based on cationically modified CNCs. ${ }^{68}$ Although the three-region pattern of flow curves were observed for some concentrations of $\mathrm{C}_{8^{-}}$and $\mathrm{C}_{12}-\mathrm{CNCs}$, similar to the pattern characteristic for unmodified CNCs (SI, Figure S14), the underlying structural changes are different for these materials. We suggest that the first shear thinning region is related to defragmentation of a network formed by small aggregates of hydrophobized CNCs. In the second region aggregates are disassembled into individual nanorods or stacks of few nanorods. At high shear rates, shear thinning is caused by the alignment of re-dispersed CNCs. 


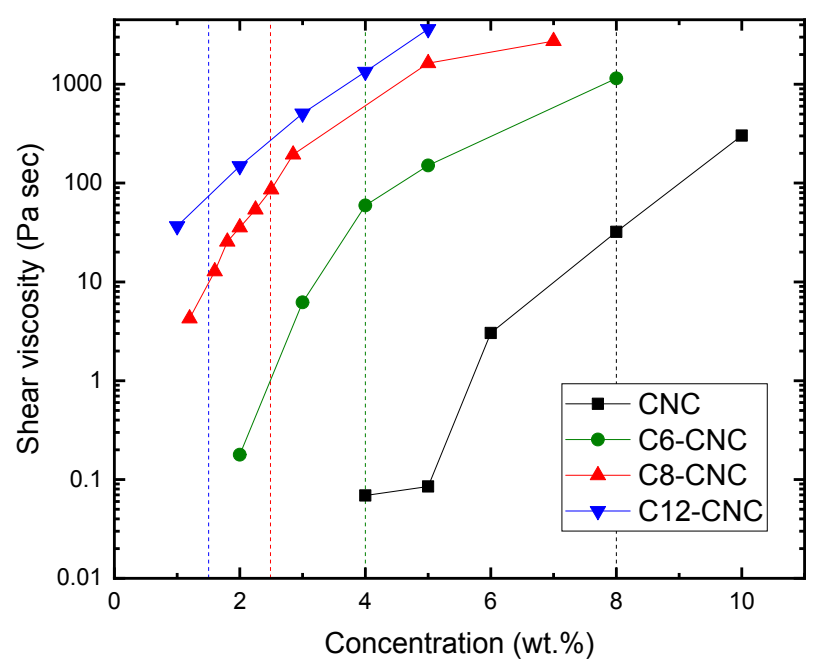

Figure 9. Dependence of steady flow viscosity of unmodified and hydrophobized CNC suspensions/gels in deionized water on CNC concentrations at shear rate of $0.1 \mathrm{~s}^{-1}$. Vertical lines indicate concentrations for forming invertible gels (color of the lines matches with color of symbols for the corresponding experimental points)

\section{Conclusions}

$\mathrm{CNCs}$ have been modified with alkylamines of different length $(\mathrm{C} 6, \mathrm{C} 8, \mathrm{C} 12)$ to vary the hydrophilic-hydrophobic balance. The properties of aqueous colloidal systems with hydrophobized CNCs have been studied particularly to assess the impact of hydrophobic effects on $\mathrm{CNC}$ association and gelation in aqueous media. We have demonstrated that hydrophobicity of modified CNCs correlated with the chain lengths of alkylamines. Although a two-stage modification process based on reductive amination led to a decrease in the content of surface sulfate half-esters, at least half of this ionic group was preserved providing a good colloidal stability of hydrophobized CNCs. Thus, the surface of modified CNCs contained both hydrophilic and hydrophobic domains. As a result, the modified CNCs are surface active nanoparticles demonstrating amphiphilic properties. However, there appeared to be no direct correlation between 
the length of alkyl chain attached to the $\mathrm{CNC}$ surface and surface activity; $\mathrm{C}_{12}-\mathrm{CNCs}$ induced smaller decrease in interfacial tension than $\mathrm{C}_{8}$-CNCs which was attributed to stronger association driven by hydrophobic effects. Self-association of hydrophobized CNCs was confirmed by SAXS and autofluorescent spectroscopy experiments. Formation of transient networks by hydrophobized CNCs due to this self-association induces changes in gelation and rheological properties of CNC suspensions. Critical concentrations of sol/gel transitions for hydrophobized CNC are significantly lower than the hydrophilic CNCs and depends on hydrophobicity of modified CNCs. Self-association of hydrophobic CNCs results in stronger, more rigid gels. Our work highlights the versatility and efficiency of modifying rheological properties of aqueous systems via self-association of hydrophobized CNCs.

\section{ASSOCIATED CONTENT}

Supporting Information. The Supporting Information is available free of charge on the ACS Publications website at DOI: solid state NMR spectra and peak deconvolution; Raman spectrum of oxidized CNCs; pictures of sessile drops on surfaces of unmodified and hydrophobized CNCs films; results of multi-channel confocal laser scanning spectroscopy; results of SAXS experiments; pictures of unmodified and hydrophobized CNC suspensions of different concentrations; rheology results for aqueous suspensions/gels; images of gels in cross-polarized light (PDF).

\section{AUTHOR INFORMATION}

Corresponding Author 


\author{
* Stephen J. Eichhorn: s.j.eichhorn@,bristol.ac.uk \\ * Rinat Nigmatullin: rn17541@,bristol.ac.uk
}

\author{
Present Addresses \\ † Departamento de Química Orgánica, Universidad de Sevilla, C/ Prof. García \\ González, 1, 41012 Sevilla, Spain; Instituto de Investigaciones Químicas (CSIC-US),
}

Avda. Américo Vespucio, 49, 41092 Sevilla, Spain

\begin{abstract}
Author Contributions
The manuscript was written through contributions of all authors led by R.N. and S.J.E. All authors have given approval to the final version of the manuscript.
\end{abstract}

\title{
Funding Sources
}

The Engineering and Physical Sciences Research Council (EPSRC) is acknowledged for provision of financial support (EP/N03340X/2, EP/N033337/1).

\section{ACKNOWLEDGMENT}

The authors gratefully acknowledge the KRÜSS Surface Science Centre (KSSC) at the University of Bristol and the Material and Chemical Characterization Facility (MC2) at the University of Bath (http://go.bath.ac.uk/mc2) for access to research equipment and assistance in this work. Dr Nick Terril and Dr Andrew Smith on beamline I22 are thanked for assistance with SAXS experiments at Diamond Light Source Ltd (experiments no. SM17580-1). Prof. Robert 
Richardson is thanked for his help with SAXS measurements on the Ganesha. The Engineering and Physical Sciences Research Council (EPSRC) is acknowledged for provision of financial support (EP/N033337/1) for J.C.M.G., J.A. and Y.Z.K. We are also grateful for UEA Faculty of Science NMR facility. V.G. would like to acknowledge the support of BBSRC Norwich Research Park Bioscience Doctoral Training Grant (BB/M011216/1).

\begin{abstract}
ABBREVIATIONS
CAC critical aggregation concentration; $\mathrm{CNC}$ cellulose nanocrystal; CNM cellulose nanomaterial; DI deionized; LVE linear viscoelastic; MCLSS multi-channel confocal laser scanning spectroscopy; SAXS small-angle X-ray scattering.
\end{abstract}

\title{
REFERENCES
}

1. Stark, W. J.; Stoessel, P. R.; Wohlleben, W.; Hafner, A. Industrial applications of nanoparticles. Chem. Soc. Rev. 2015, 44 (16), 5793-5805.

2. Heiligtag, F. J.; Niederberger, M. The fascinating world of nanoparticle research. Mater. Today 2013, 16 (7), 262-271.

3. Jutz, G.; Böker, A. Bionanoparticles as functional macromolecular building blocks - A new class of nanomaterials. Polymer 2011, 52 (2), 211-232.

4. Lin, N.; Huang, J.; Dufresne, A. Preparation, properties and applications of polysaccharide nanocrystals in advanced functional nanomaterials: a review. Nanoscale 2012, 4 (11), 3274-3294. 
5. Klemm, D.; Kramer, F.; Moritz, S.; Lindström, T.; Ankerfors, M.; Gray, D.; Dorris, A. Nanocelluloses: A new family of nature-based materials. Angew. Chem., Int. Ed. 2011, 50 (24), $5438-5466$.

6. Abitbol, T.; Rivkin, A.; Cao, Y.; Nevo, Y.; Abraham, E.; Ben-Shalom, T.; Lapidot, S.; Shoseyov, O. Nanocellulose, a tiny fiber with huge applications. Curr. Opin. Biotechn. 2016, 39, 76-88.

7. Gang, O. Nanoparticle assembly: from fundamentals to applications: concluding remarks. Faraday Discuss. 2016, 186, 529-537.

8. Grzelczak, M.; Vermant, J.; Furst, E. M.; Liz-Marzán, L. M., Directed self-assembly of nanoparticles. ACS Nano 2010, 4 (7), 3591-3605.

9. Li, F.; Lu, J.; Kong, X.; Hyeon, T.; Ling, D. Dynamic nanoparticle assemblies for biomedical applications. Adv. Mater. 2017, 29 (14), 1605897.

10. Gray, D.; Mu, X. Chiral nematic structure of cellulose nanocrystal suspensions and films; Polarized light and atomic force microscopy. Materials 2015, 8 (11), 5427.

11. Capron, I.; Rojas, O. J.; Bordes, R. Behavior of nanocelluloses at interfaces. Curr. Opin. Colloid Interface Sci. 2017, 29, 83-95.

12. Eyley, S.; Thielemans, W. Surface modification of cellulose nanocrystals. Nanoscale 2014, $6(14), 7764-7779$.

13. Habibi, Y. Key advances in the chemical modification of nanocelluloses. Chem. Soc. Rev. 2014, 43 (5), 1519-1542. 
14. Goussé, C.; Chanzy, H.; Excoffier, G.; Soubeyrand, L.; Fleury, E., Stable suspensions of partially silylated cellulose whiskers dispersed in organic solvents. Polymer 2002, 43 (9), $2645-2651$.

15. Goussé, C.; Chanzy, H.; Cerrada, M. L.; Fleury, E. Surface silylation of cellulose microfibrils: preparation and rheological properties. Polymer 2004, 45 (5), 1569-1575.

16. Andresen, M.; Johansson, L.-S.; Tanem, B. S.; Stenius, P. Properties and characterization of hydrophobized microfibrillated cellulose. Cellulose 2006, 13 (6), 665-677.

17. Cunha, A. G.; Mougel, J.-B.; Cathala, B.; Berglund, L. A.; Capron, I. Preparation of double pickering emulsions stabilized by chemically tailored nanocelluloses. Langmuir 2014, 30 (31), 9327-9335.

18. Sojoudiasli, H.; Heuzey, M.-C.; Carreau, P. J.; Riedl, B., Rheological behavior of suspensions of modified and unmodified cellulose nanocrystals in dimethyl sulfoxide. Rheol. Acta 2017, $56(7), 673-682$.

19. Siqueira, G.; Bras, J.; Dufresne, A., Cellulose whiskers versus microfibrils: Influence of the nature of the nanoparticle and its surface functionalization on the thermal and mechanical properties of nanocomposites. Biomacromolecules 2009, 10 (2), 425-432.

20. Shang, W.; Huang, J.; Luo, H.; Chang, P. R.; Feng, J.; Xie, G., Hydrophobic modification of cellulose nanocrystal via covalently grafting of castor oil. Cellulose 2013, 20 (1), 179-190.

21. Lee, Y. R.; Park, D.; Choi, S. K.; Kim, M.; Baek, H. S.; Nam, J.; Chung, C. B.; Osuji, C. O.; Kim, J. W. Smart cellulose nanofluids produced by tunable hydrophobic association of polymer-grafted cellulose nanocrystals. ACS Appl. Mater. Interfaces 2017, 9 (36), 31095-31101. 
22. Yoo, Y.; Youngblood, J. P. Green one-pot synthesis of surface hydrophobized cellulose nanocrystals in aqueous medium. ACS Sustainable Chem. Eng. 2016, 4 (7), 3927-3938.

23. Shimizu, M.; Saito, T.; Fukuzumi, H.; Isogai, A. Hydrophobic, ductile, and transparent nanocellulose films with quaternary alkylammonium carboxylates on nanofibril surfaces. Biomacromolecules 2014, 15 (11), 4320-4325.

24. Salajkova, M.; Berglund, L. A.; Zhou, Q., Hydrophobic cellulose nanocrystals modified with quaternary ammonium salts. J. Mater. Chem. 2012, 22 (37), 19798-19805.

25. Shimizu, M.; Saito, T.; Isogai, A., Bulky quaternary alkylammonium counterions enhance the nanodispersibility of 2,2,6,6-tetramethylpiperidine-1-oxyl-oxidized cellulose in diverse solvents. Biomacromolecules 2014, 15 (5), 1904-1909.

26. Ansari, F.; Salajková, M.; Zhou, Q.; Berglund, L. A., Strong surface treatment effects on reinforcement efficiency in biocomposites based on cellulose nanocrystals in oly(vinyl acetate) matrix. Biomacromolecules 2015, 16 (12), 3916-3924.

27. Cervin, N. T.; Johansson, E.; Benjamins, J.-W.; Wågberg, L. Mechanisms behind the stabilizing action of cellulose nanofibrils in wet-stable cellulose foams. Biomacromolecules 2015, $16(3), 822-831$.

28. Kedzior, S. A.; Marway, H. S.; Cranston, E. D. Tailoring cellulose nanocrystal and surfactant behavior in miniemulsion polymerization. Macromolecules 2017, 50 (7), 2645-2655.

29. Saidane, D.; Perrin, E.; Cherhal, F.; Guellec, F.; Capron, I. Some modification of cellulose nanocrystals for functional Pickering emulsions. Philos. Trans. A Math. Phys. Eng. Sci. 2016, 374 (2072), 20150139. 
30. Wei, Z.; Sinko, R.; Keten, S.; Luijten, E. Effect of surface modification on water adsorption and interfacial mechanics of cellulose nanocrystals. ACS Appl. Mater. Interfaces 2018, 10 (9), $8349-8358$.

31. Hu, Z.; Ballinger, S.; Pelton, R.; Cranston, E. D. Surfactant-enhanced cellulose nanocrystal Pickering emulsions. J. Colloid Interface Sci. 2015, 439, 139-148.

32. Fox, D. M.; Rodriguez, R. S.; Devilbiss, M. N.; Woodcock, J.; Davis, C. S.; Sinko, R.; Keten, S.; Gilman, J. W. Simultaneously tailoring surface energies and thermal stabilities of cellulose nanocrystals using ion exchange: Effects on polymer composite properties for transportation, infrastructure, and renewable energy applications. ACS Appl. Mater. Interfaces 2016, 8 (40), 27270-27281.

33. Dhar, N.; Au, D.; Berry, R. C.; Tam, K. C. Interactions of nanocrystalline cellulose with an oppositely charged surfactant in aqueous medium. Colloids Surf., A 2012, 415, 310-319.

34. Brinatti, C.; uang, J.; Berry, R. M.; Tam, K. C.; Loh, W. Structural and energetic studies on the interaction of cationic surfactants and cellulose nanocrystals. Langmuir 2016, 32 (3), 689-698.

35. Dash, R.; Elder, T.; Ragauskas, A. J. Grafting of model primary amine compounds to cellulose nanowhiskers through periodate oxidation. Cellulose 2012, 19 (6), 2069-2079.

36. Ojala, J.; Sirviö, J. A.; Liimatainen, H., Nanoparticle emulsifiers based on bifunctionalized cellulose nanocrystals as marine diesel oil-water emulsion stabilizers. Chem. Eng. J. 2016, 288, 312-320. 
37. Visanko, M.; Liimatainen, H.; Sirviö, J. A.; Heiskanen, J. P.; Niinimäki, J.; Hormi, O. Amphiphilic cellulose nanocrystals from acid-free oxidative treatment: Physicochemical characteristics and use as an oil-water stabilizer. Biomacromolecules 2014, 15 (7), 2769-2775.

38. Tang, C.; Spinney, S. B.; Shi, Z.; Tang, J.; Peng, B.; Luo, J.; Tam, K. C., Amphiphilic cellulose nanocrystals for enhanced Pickering emulsion stabilization. Langmuir 2018, 34 (43), 12897-12905.

39. Hu, Z.; Berry, R. M.; Pelton, R.; Cranston, E. D. One-pot water-based hydrophobic surface modification of cellulose nanocrystals using plant polyphenols. ACS Sustainable Chem. Eng. 2017, 5 (6), 5018-5026.

40. Lee, K.-Y.; Aitomäki, Y.; Berglund, L. A.; Oksman, K.; Bismarck, A. On the use of nanocellulose as reinforcement in polymer matrix composites. Compos. Sci. Technol. 2014, 105, $15-27$.

41. Chen, J.; Lin, N.; Huang, J.; Dufresne, A. Highly alkynyl-functionalization of cellulose nanocrystals and advanced nanocomposites thereof via click chemistry. Polymer Chem. 2015, 6 (24), 4385-4395.

42. Gan, L.; Liao, J.; Lin, N.; Hu, C.; Wang, H.; Huang, J. Focus on gradientwise control of the surface acetylation of cellulose nanocrystals to optimize mechanical reinforcement for hydrophobic polyester-based nanocomposites. ACS Omega 2017, 2 (8), 4725-4736.

43. Peng, S. X.; Shrestha, S.; Yoo, Y.; Youngblood, J. P. Enhanced dispersion and properties of a two-component epoxy nanocomposite using surface modified cellulose nanocrystals. Polymer 2017, 112, 359-368. 
44. Palange, C.; Johns, M. A.; Scurr, D. J.; Phipps, J. S.; Eichhorn, S. J. The effect of the dispersion of microfibrillated cellulose on the mechanical properties of melt-compounded polypropylene-polyethylene copolymer. Cellulose 2019, 26 (18), 9645-9659.

45. Chassenieux, C.; Nicolai, T.; Benyahia, L. Rheology of associative polymer solutions. Current Opinion in Colloid \& Interface Science 2011, 16 (1), 18-26.

46. Winnik, M. A.; Yekta, A., Associative polymers in aqueous solution. Curr. Opin. Colloid Interface Sci. 1997, 2 (4), 424-436.

47. Wang, J.; Benyahia, L.; Chassenieux, C.; Tassin, J.-F.; Nicolai, T., Shear-induced gelation of associative polyelectrolytes. Polymer 2010, 51 (9), 1964-1971.

48. Nigmatullin, R.; Harniman, R.; Gabrielli, V.; Muñoz-García, J. C.; Khimyak, Y. Z.; Angulo, J.; Eichhorn, S. J. Mechanically robust gels formed from hydrophobized cellulose nanocrystals. ACS Appl. Mater. Interfaces 2018, 10 (23), 19318-19322.

49. Nigmatullin, R.; Gabrielli, V.; Muñoz-García, J. C.; Lewandowska, A. E.; Harniman, R.; Khimyak, Y. Z.; Angulo, J.; Eichhorn, S. J. Thermosensitive supramolecular and colloidal hydrogels via self-assembly modulated by hydrophobized cellulose nanocrystals. Cellulose $\mathbf{2 0 1 9}$, $26(1), 529-542$.

50. Larsson, P. T.; Wickholm, K.; Iversen, T. A CP/MAS13C NMR investigation of molecular ordering in celluloses. Carbohydr. Res. 1997, 302 (1), 19-25.

51. Abitbol, T.; Kloser, E.; Gray, D. G. Estimation of the surface sulfur content of cellulose nanocrystals prepared by sulfuric acid hydrolysis. Cellulose 2013, 20 (2), 785-794. 
52. Schmitt, J.; Calabrese, V.; da Silva, M. A.; Lindhoud, S.; Alfredsson, V.; Scott, J. L.; Edler, K. J. TEMPO-oxidised cellulose nanofibrils; probing the mechanisms of gelation via small angle X-ray scattering. Phys. Chem. Chem. Phys. 2018, 20 (23), 16012-16020.

53. Foster, E. J.; Moon, R. J.; Agarwal, U. P.; Bortner, M. J.; Bras, J.; Camarero-Espinosa, S.; Chan, K. J.; Clift, M. J. D.; Cranston, E. D.; Eichhorn, S. J.; Fox, D. M.; Hamad, W. Y.; Heux, L.; Jean, B.; Korey, M.; Nieh, W.; Ong, K. J.; Reid, M. S.; Renneckar, S.; Roberts, R.; Shatkin, J. A.; Simonsen, J.; Stinson-Bagby, K.; Wanasekara, N.; Youngblood, J. Current characterization methods for cellulose nanomaterials. Chem. Soc. Rev. 2018, 47, 2609-2679

54. Oguzlu, H.; Danumah, C.; Boluk, Y. Colloidal behavior of aqueous cellulose nanocrystal suspensions. Curr. Opin. Colloid Interface Sci. 2017, 29, 46-56.

55. Reid, M. S.; Villalobos, M.; Cranston, E. D. Benchmarking cellulose nanocrystals: From the laboratory to industrial production. Langmuir 2017, 33 (7), 1583-1598.

56. Chandar, P.; Somasundaran, P.; Turro, N. J. Fluorescence probe studies on the structure of the adsorbed layer of dodecyl sulfate at the alumina—water interface. J. Colloid Interface Sci. 1987, $117(1), 31-46$.

57. Misra, P. K.; Somasundaran, P. Fluorescence probing of the surfactant assemblies in solutions and at solid-liquid interfaces. In Interfacial Processes and Molecular Aggregation of Surfactants, Narayanan, R., Ed. Springer Berlin Heidelberg: Berlin, Heidelberg, 2008; pp 143-188.

58. Piñeiro, L.; Novo, M.; Al-Soufi, W. Fluorescence emission of pyrene in surfactant solutions. Adv. Colloid Interface Sci. 2015, 215, 1-12. 
59. Johns, M. A.; Lewandowska, A. E.; Eichhorn, S. J. Rapid determination of the distribution of cellulose nanomaterial aggregates in composites enabled by multi-channel spectral confocal microscopy. Microsc. Microanal. 2019, 25(3), 682-689.

60. Ureña-Benavides, E. E.; Ao, G.; Davis, V. A.; Kitchens, C. L. Rheology and phase behavior of lyotropic cellulose nanocrystal suspensions. Macromolecules 2011, 44 (22), 8990-8998.

61. Shafiei-Sabet, S.; Hamad, W. Y.; Hatzikiriakos, S. G. Rheology of nanocrystalline cellulose aqueous suspensions. Langmuir 2012, 28 (49), 17124-17133.

62. Bercea, M.; Navard, P. Shear Dynamics of Aqueous Suspensions of Cellulose Whiskers. Macromolecules 2000, 33 (16), 6011-6016.

63. Xu, Y.; Atrens, A. D.; Stokes, J. R. Rheology and microstructure of aqueous suspensions of nanocrystalline cellulose rods. J. Colloid Interface Sci. 2017, 496, 130-140.

64. Picout, D. R.; Ross-Murphy, S. B. Rheology of biopolymer solutions and gels Sci. World J., 2003, 3, 105-121.

65. Phan-Xuan, T.; Thuresson, A.; Skepö, M.; Labrador, A.; Bordes, R.; Matic, A. Aggregation behavior of aqueous cellulose nanocrystals: the effect of inorganic salts. Cellulose 2016, 23 (6), 3653-3663.

66. Hongladarom, K.; Burghardt, W. R. Molecular orientation, "Region I" shear thinning and the cholesteric phase in aqueous hydroxypropylcellulose under shear. Rheol. Acta 1998, 37 (1), 46-53. 
67. Mewis, J.; Moldenaers, P. Rheology of polymeric liquid crystals. Curr. Opin. Colloid Interface Sci. 1996, 1 (4), 466-471.

68. Hasani, M.; Cranston, E. D.; Westman, G.; Gray, D. G., Cationic surface functionalization of cellulose nanocrystals. Soft Matter 2008, 4 (11), 2238-2244. 
$82 \times 37 m m(149 \times 149$ DPI $)$

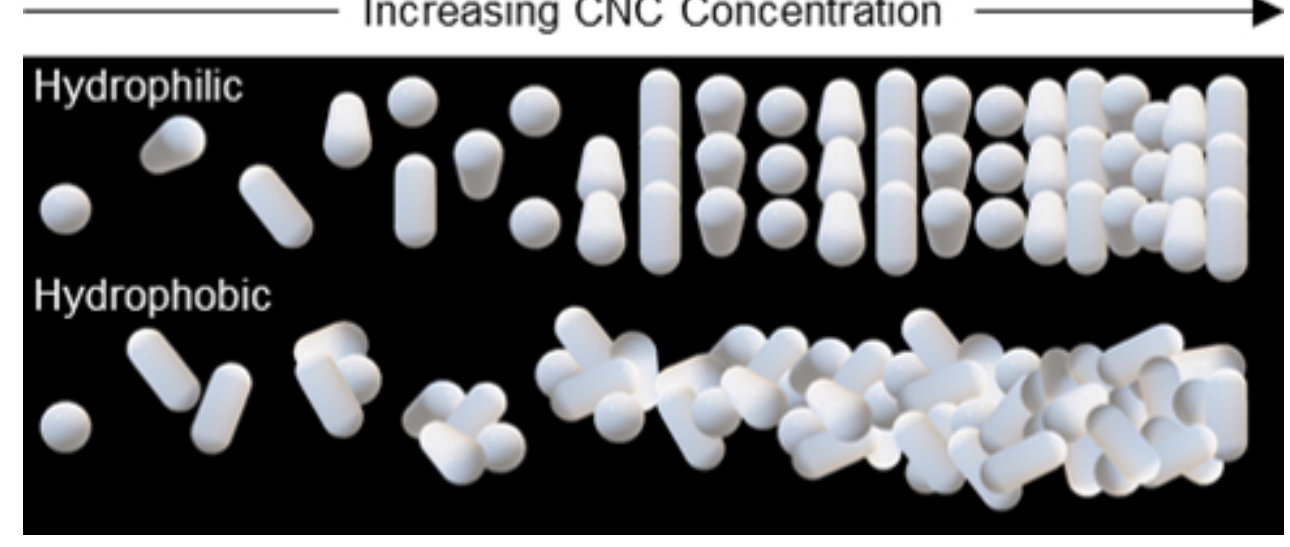

